\title{
A Numerical Study of Baroclinic Instability at Large Supercriticality*
}

\author{
PATRICE KLEIN \\ Laboratoire d'Océanographie Physique, Faculté des Sciences, Université de Bretagne Occidentale, 29287 - Brest, France \\ JOSEPH PEDLOSKY \\ Woods Hole Oceanographic Institution, Woods Hole, MA 02543
}

(Manuscript received 3 October 1985, in final form 17 January 1986)

\begin{abstract}
A series of numerical integrations of the two-layer quasi-geostrophic model were carried out to investigate the nonlinear dynamics of baroclinically unstable waves at supercriticalities of $O(1)$. The results extend and are contrasted with the results of weakly nonlinear theory valid only for small supercriticality.

Particular attention is paid to that sector of parameter space in which the transition from regular to aperiodic behavior is observed for weakly nonlinear waves. It is found that aperiodic, chaotic behavior extends to parameter domains of higher dissipation as a consequence of finite amplitude effects as the supercriticality increases.

Sensitive dependence on parameters remains a hallmark of the system as intervals of chaotic, periodic and steady solutions are observed. For the supercriticality of $\mathrm{O}(1)$ a new stable periodic vacillation is observed.

As the supercriticality is increased the system appears to "stiffen" nonlinearly, e.g., wave amplitudes in the steady state are smaller than predicted by weakly nonlinear scaling arguments. This stiffening can be explained in terms of the dynamics of a truncated system. However, the truncated system appears always to overestimate the domain of chaotic behavior since it misrepresents a subtle effect of the higher harmonics on the process of wave-mean flow interaction.

At much higher supercriticality (e.g., four times critical) where many waves are unstable, it is found that the linearly most unstable wave gives way to a longer, less unstable wave which comes to dominate the solution in qualitative agreement with the predictions of weakly nonlinear theory.

In all cases, in order to separate truly nonlinear effects from the parametric variations already present in asymptotic weakly nonlinear theory calculations described here were done for increasing supercriticality at a fixed value of $\gamma=$ (linear $e$-folding time)/(spinup time) the numerator of which decreases with increasing supercriticality. Hence in our presentation at larger supercriticality the friction is also greater.
\end{abstract}

\section{Introduction}

\section{a. Preface}

The state of flow of a spontaneously unstable baroclinic system will lie between two extreme limits. One limit occurs for only slightly unstable systems in which the amplitude of the fluctuations resulting from the instability is very small, in principle infinitesimal in the limit of vanishing supercriticality. The other limit can be described as fully developed turbulence in which the amplitude of the fluctuations is so great that the energy in the random fluctuation field dominates the energy spectrum of the field of motion.

Both of these limiting cases have received a considerable amount of attention by theorists and although a great number of important and interesting problems remain to be solved it may safely be said that these two extremes are tolerably well understood. In each case

\footnotetext{
* Woods Hole Institute of Oceanography Contribution number 6085 .
}

the nature of the appropriate limit involved in approaching the characterization of the extreme state introduces mathematical and physical simplifications that may be exploited.

In the case of fully developed geostrophic turbulence the idealization of the flow as homogeneous, with an absence of background flow structure simplifies the interpretation and calculation of the flow (e.g., Rhines, 1979; Salmon, 1982). Even in this limit the expectations of complete structural incoherence may be confounded (McWilliams, 1984) but, inarguably the simplifications associated with the absence of a background structured current are exploited.

At the other limit, i.e., of small amplitude fluctuation motion, the methods of weakly nonlinear theory may be used to describe the resulting motion. Studies by Drazin (1970, 1972), Hart (1972, 1973, 1981), Pedlosky $(1970,1972,1981)$ and Pedlosky and Frenzen (1980) have each exploited the smallness of the fluctuation motion to use asymptotic methods to derive simplified equations to describe a field of flow containing a relatively small number of spatial modes. We shall review 
certain salient features of the dynamics in this limit shortly but it is appropriate to remark here that even in this limit the dynamics can be quite complex in its behavior in the time domain.

In fact, many systems of flow of interest in meteorology and oceanography lie in some intermediate region between these extremes in a state in which neither the conceptual simplifications of weakly nonlinear theory nor of fully developed turbulence apply. Therefore it seemed to us to be useful to initiate a program of study which started from one fairly well understood limit and systematically advanced into the terra incognita of the intermediate zone. Our experience studying weakly nonlinear theory naturally predisposes us to start from the small amplitude, weakly nonlinear end of the interval. We report in this paper some interesting initial results of our investigation into the dynamics of unstable baroclinic flows when the appropriate measures of the supercriticality and of the amplitude of the fluctuations are each of $O(1)$.

Studies of weakly nonlinear theory reveal that the presence of the beta effect, horizontal shear, or asymmetric application of boundary conditions (e.g., the presence of one free and one rigid horizontal boundary, etc.) render even the weakly nonlinear problem quite complex. Therefore we have chosen to concentrate on the simplest and conceptually "cleanest" model, e.g., two-layer flow with only vertical shear on the $f$-plane in an infinite channel bounded above and below by rigid boundaries on which Ekman layers provide the sole source of frictional dissipation. This is precisely the physical model to which the asymptotic theories of Pedlosky (1972) and Pedlosky and Frenzen (1980) apply in the limit of weak supercriticality. The interesting range of behavior found even in the weakly nonlinear limit seemed adequate reason to choose this highly idealized model. We hasten to stress that our proximate goal is not the simulation of a realistic atmospheric or oceanic flow but rather the development of a description and understanding of a process of undoubted geophysical importance in a somewhat more realistic range of nonlinearity.

Our first goal is to contrast the results of our calculations with the results of weakly nonlinear theory as the measure of the flow's supercriticality is increased from a very small value to a value of order unity. The method of our investigation is, with a single exception, entirely numerical. The precise numerical method is described below but we wish to quickly acknowledge the intrinsic difficulties and uncertainties associated with such an approach and hence we acknowledge the inherently tentative quality of some of our conclusions about which we will be more specific at appropriate points within the paper.

There have, of course, already been some very interesting numerical studies that are in some respects similar to ours, e.g., Boville $(1980,1982)$. However, rather than attempting to simulate a particular vacil- lation phenomenon in a restricted parameter range, we have instead attempted to cover a broader parameter range, at least initially, to describe the metamorphosis of the structure of the flow as the supercriticality is increased. From this point of view it will prove useful to quickly review certain features of weakly nonlinear theory.

\section{b. Review of weakly nonlinear theory}

The finite amplitude evolution of a weakly unstable, weakly nonlinear wave has been described by Pedlosky and Frenzen (1980). The theory describes the evolution of a single baroclinic wave in a two-layer, quasi-geostrophic model in which the dissipation is produced by Ekman layers on the upper and lower solid surfaces. The basic flow consists of a uniform zonal motion in the $x$-direction with nondimensional velocity $U_{1}$ in the upper layer and $U_{2}$ in the lower layer. The motion is contained in a channel of width $L$ uniformly rotating with angular velocity $\Omega$ about the $z$-axis. The nondimensional cross-stream coordinate $y$ ranges from 0 to 1. The depth of each layer in the absence of motion is $D$ and the (statically stable) density difference between the layers is $\Delta \rho$. The density in the lower layer is $\rho$ $+\Delta \rho$ while it is $\rho$ in the upper layer. Both $\rho$ and $\Delta \rho$ are constant and $0<\Delta \rho / \rho \ll 1$. The kinematic viscosity in each layer is $\nu$ and the effect of dissipation in the quasi-geostrophic system is measured by the parameter

$$
r=\frac{2(\nu \Omega)^{1 / 2} L}{U D}
$$

where $U$ is the scale used to relate the dimensionless to the dimensional horizontal velocity. The perturbation, at lowest order, to the basic flow is described by a geostrophic streamfunction which is barotropic and has the form:

$$
\psi=\frac{\Delta^{1 / 2}\left(U_{1}-U_{2}\right)}{2 a \pi} A(t) e^{i k x} \sin \pi y+*,
$$

where the asterisk represents the complex conjugate of the preceding expression and where the development time $t$ is scaled by the linear $e$-folding time. The key parameters of the problem are

$$
\begin{aligned}
& F=4 \Omega^{2} L^{2} /\left(g \frac{\Delta \rho}{\rho} D\right) \\
& \gamma=r / 2 \sigma .
\end{aligned}
$$

In (3) $\sigma$ is the nondimensional growth rate, i.e.,

$$
\sigma=\frac{k\left(U_{1}-U_{2}\right)}{2 a} \Delta^{1 / 2}
$$

where $a^{2}=k^{2}+\pi^{2}$ and $\Delta$ is the supercriticality, i.e., $\Delta$ is the difference between the value of $F$ and its critical value for linear instability, viz: 


$$
F_{c}=\frac{a^{2}}{2}+\frac{r^{2} a^{2}}{2 k^{2}\left(U_{1}-U_{2}\right)^{2}}
$$

so that

$$
\Delta \equiv F-F_{c}
$$

The mean flow is chosen, by a Galilean transformation to have $U_{1}=-U_{2}$ so that the wave is nonpropagating. Dimensional times and rates are obtained from their nondimensional counterparts by use of the time scale $(L / U \sigma)$.

It can be shown, Pedlosky and Frenzen (1980), that $A$ satisfies

$$
\frac{d^{2} A}{d t^{2}}+\frac{3 \gamma}{2} \frac{d A}{d t}-A+A \int_{0}^{1} d y \sin 2 \pi y \frac{\partial^{2} \psi}{\partial y^{2}}=0
$$

where $\psi(y, t)$ is the correction to the streamfunction for the zonal flow which satisfies

$\frac{\partial}{\partial t}\left(\frac{\partial^{2} \psi}{\partial y^{2}}-a^{2} \psi\right)+\gamma \frac{\partial^{2} \psi}{\partial y^{2}}=\left(\frac{d}{d t}|A|^{2}+2 \gamma|A|^{2}\right) \sin 2 \pi y$

with

$$
\frac{\partial \psi}{\partial y}=0 \text { on } \quad y=0,1
$$

For all values of $a^{2}$ and $\gamma$ two steady solutions exist, which aside from a constant and irrelevant phase factor may be written as $A= \pm 1$. Note that this implies that the actual amplitude of the disturbance, if steady, increases as $\Delta^{1 / 2}$ in weakly nonlinear theory. For most values of $\gamma$ this steady state is stable to infinitesimal perturbations but this stability is practically irrelevant to the initial value problem whose initial conditions lie distant from this end state.

A sequence of numerical integrations of (7) and (8) instead reveal the following. For small enough values of $\gamma$ (e.g., for $a^{2}=2 \pi^{2}$, for $\gamma \leqslant 0.1295$ ) a simple, perpetually periodic solution is obtained in which $A$ oscillates about zero with an amplitude $\sim 1.6$. As $\gamma$ increases, an interval of period doubling occurs for 0.1295 $\leqslant \gamma \leqslant 0.1307$ during which oscillations of doubled period become the stable solutions. Again the oscillation passes through zero but a return to any particular amplitude peak requires increasingly many cycles as $\gamma$ increases to the upper limit of the interval. Beyond $\gamma$ $\sim 0.1307$ chaotic behavior is observed until $\gamma$ reaches a critical value, dependent on $a^{2}$. For $a^{2}=2 \pi^{2}$ this critical value, $\gamma_{c}$, was observed to be $\sim 0.18$. For $\gamma$ $>\gamma_{c}$ the solution becomes captured by one of the stable steady states although a considerable period of chaotic behavior can occur before the wave amplitude becomes steady (mainly when $\gamma$ is close to $\gamma_{c}$ ). Within the interval of chaotic behavior isolated intervals in $\gamma$ of periodic behavior are observed. The periodic oscillations in this interval are qualitatively different from those involved at low $\gamma$, for these new periodic solutions for $A$, while passing through zero, generally contain a seg- ment in time in which an oscillation about the steady solution occurs during which the minimum amplitude remains greater than zero.

If $\psi(y, t)$ is expanded in the series

$$
\psi=\sum_{n=1}^{\infty} \psi_{0 n} \cos n \pi y
$$

which automatically satisfies (9), and the sum is arbitrarily truncated after the first term, i.e., if the representation for $\psi$ is simply taken as $\psi=\psi_{01} \cos \pi y$ then $\psi_{01}$ and $A$ would satisfy

$$
\begin{gathered}
\frac{d^{2} A}{d t^{2}}+\frac{3 \gamma}{2} \frac{d A}{d t}-A-\frac{4 \pi}{3} A \psi_{01}=0 \\
\frac{d \psi_{01}}{d t}+\frac{\gamma \pi^{2}}{a^{2}+\pi^{2}} \psi_{01}=\left(\frac{d A^{2}}{d t}+2 \gamma A^{2}\right) \frac{-8}{3 \pi\left(\pi^{2}+a^{2}\right)} .
\end{gathered}
$$

This system can be put in the form of the well-studied Lorenz system

$$
\begin{aligned}
& \frac{d \eta}{d \tau}=\mu(\xi-\eta) \\
& \frac{d \xi}{d \tau}=R \eta-\xi-\eta \zeta \\
& \frac{d \zeta}{d \tau}=\xi \eta-\beta \zeta
\end{aligned}
$$

with the transformation

$$
\begin{aligned}
\mu & =2 \\
\tau & =\frac{\gamma}{2} t \\
\beta & =\frac{2 \pi^{2}}{a^{2}+\pi^{2}} \\
R & =\frac{2}{\gamma^{2}}\left(1+\gamma^{2} / 2\right) \\
\eta & =\frac{16}{3 \gamma\left(\pi^{2}+a^{2}\right)^{1 / 2}} A \\
\xi & =\eta+\frac{1}{2} \frac{\partial \eta}{\partial \tau} \\
\zeta & =\frac{8 \pi}{3 \gamma^{2}} \psi_{01} \cdot
\end{aligned}
$$$$
(14 a, b, c, d, e, f, g)
$$

The Lorenz equations are known to possess a rich and complex behavior (e.g., see the monograph of Sparrow, 1982, for a review) so that it is, perhaps, no surprise to find the full system (7), (8), (9) displaying a similarly complex and sensitive behavior. For meteorological and oceanographic purposes, clearly the most striking aspect of this behavior is the appearance of chaotic, aperiodic and thus essentially unpredictable motion in such low order systems. Even if the range 
of $\gamma$ in which the chaotic behavior occurs is relatively small, $0.13 \leqslant \gamma \leqslant 0.18$, this corresponds to a geophysically interesting interval in which the viscous decay time is a few times longer than the $e$-folding time of linear instability. It remains to be seen what actual connection there is between the Lorenz-like chaotic behavior and geophysical indeterminacy but the phenomenon is too striking to ignore. Nevertheless a real, and in our experience, often expressed concern is whether this complex behavior, including aperiodic motion, is somehow the consequence of a dynamical delicacy expected of weakly nonlinear theory and may not the "Lorenzian" behavior disappear for more robust fluctuations which would occur at higher supercriticality, i.e., $\Delta=\mathrm{O}(1)$ for which (7), (8) are no longer valid. Naturally this is one of the first questions we investigate in the calculations to be reported on below.

A related question which has been addressed by weakly nonlinear theory is the nature by which the wavenumber of the realized finite amplitude disturbance is selected by the dynamics. Although the single wave state governed by (7) and (8) is a consistent state it requires that initially only the wave with that wavenumber be present. Hart (1981) and Pedlosky (1981) both employed weakly nonlinear theory to describe the process of competition between waves of different wavenumbers by which the most unstable wave according to linear theory could, under appropriate parametric situations, be suppressed as time goes by in favor of a longer, unstable wave with a lower growth rate. The process responsible for this selection preference of the longer wave rests in the nature of the wavemean flow interaction, a process which is dominant for flows initially without horizontal shear at low supercriticality. Thus an additional question of great interest is whether the same shift to longer wavelength will occur if the supercriticality is (i) large enough to allow several unstable waves and (ii) the most unstable wave according to linear theory is not the longer wave whose ultimate supremacy is predicted by weakly nonlinear theory.

\section{c. The equations}

To examine the nature of the nonlinear dynamics of baroclinic waves when the supercriticality is no longer small requires us to abandon the amplitude equations (7) and (8). We therefore return to the full quasi-geostrophic equations appropriate for the twolayer model (Pedlosky, 1979). Using $U, L, L / U, U L$, for the scales of velocity, length, time, and geostrophic streamfunction, the nonlinear equations for the disturbance fields $\psi_{n}(x, y, t), n=1,2$ may be written:

$$
\begin{aligned}
\frac{\partial q_{n}}{\partial t}+U_{n} \frac{\partial q_{n}}{\partial x} & -\frac{\partial \psi_{n}}{\partial x} F(-1)^{n}\left(U_{1}-U_{2}\right) \\
& +J\left(\psi_{n}, q_{n}\right)=\frac{-r}{2} \nabla^{2} \psi_{n}, \quad n=1,2
\end{aligned}
$$

where the perturbation potential vorticity,

$$
q_{n} \equiv \nabla^{2} \psi_{n}+(-1)^{n} F\left(\psi_{1}-\psi_{2}\right),
$$

and where $F$ and $r$ have been previously defined. The total streamfunction is simply $\psi_{n}-U_{n} y$ and (15) will describe the evolution of the disturbance placed on the initial flow which has vertical but no horizontal shear. It proves convenient to let $U_{1}=-U_{2}=U_{s} / 2$.

On the boundaries $y=0,1$ the velocity in the $y$ direction must vanish and this requires:

$$
\partial \psi_{n} / \partial x=0, \quad y=0,1
$$

The disturbance field $\psi_{n}$ may contain a component, $\Psi_{n}(y, t)$ which is independent of $x$. This component represents a correction to the basic flow $U_{n}$ and (16) is an empty condition for this component to which instead the condition

$$
\partial \Psi_{n} / \partial y=0, \quad y=0,1
$$

is applied, as described by Pedlosky (1970).

At $t=0$ a wavelike disturbance is placed on the flow and its evolution is computed numerically by means of the method described below.

The explicit parameters in (15) are $F$ and $r$ whereas in the weakly nonlinear theory they are $F$ and $\gamma$. The parameter $\gamma$ measures the dissipation time scale with respect to the development time of the wave amplitude. In weakly nonlinear theory the development process can be isolated from the advective processes. For supercriticalities of $\mathrm{O}(1)$ this can no longer be done and $r$, which measures the dissipation time scale against the advective scale is the appropriate measure of friction. However, were we to hold $r$ fixed and increase the supercriticality, i.e., increase $F$, it would become extremely difficult to distinguish between the effects of enhanced nonlinearity which would occur since the amplitude increases with supercriticality, and the effect of simply increasing the linear growth rate which also increases with $\Delta$. That is, for fixed $r$ increasing $\Delta$ would decrease $\gamma[(3)$, (4)]. That might change the behavior of the wave evolution, say, from steady to chaotic solely on the basis of effects already present in weakly nonlinear theory. In fact one principal attribute of the wave amplitude dynamics for small supercriticality is its extreme sensitivity to the value of $\gamma$ (Pedlosky and Frenzen, 1980). Therefore in an attempt to focus on the new effects of nonlinearity the numerical calculations to be described were conducted in the parameter space of $F$ and $r$ along lines of constant $\gamma$ :

$$
\gamma=\frac{r}{2 \sigma}, \quad \sigma=\frac{k U_{s}}{2 a}\left(F-F_{c}\right)^{1 / 2} .
$$

Since several wavenumbers are present in the calculation we choose to define $\sigma$ (i.e., $a, k$ and $F_{c}$ ) in terms of the fundamental wave in our spectral expansion (see below). Except for a single experiment described below this wave is also the most unstable. This strategy of 
exploration appeared to us to be the most convenient way to contrast the behavior of the system for $O(1)$ supercriticality to the behavior for $\Delta \ll 1$.

\section{d. Principal results}

The detailed results of roughly thirty numerical experiments will be discussed in following sections. Here we present a short synopsis of our conclusions.

Pedlosky and Frenzen (1980) expressed their despair in the fact that the eighty experiments they conducted with the system (7), (8) were obviously insufficient to fully describe the parameter dependence of the solution for the wave amplitude on $\gamma$. For larger supercriticality it can be appreciated that the situation becomes even more difficult for two obvious reasons. First, the parameter space is now three $(\Delta, \gamma, a)$ dimensional rather than two dimensional $(0, \gamma, a)$ as in the limit $\Delta \rightarrow 0$. Second, the potential of greater interaction between higher harmonics of the solution and the fundamental, (interactions which are ignorable as nonresonant and negligible for small $\Delta$ ) means that the dependence of the solution on the initial values of the spectrum of higher harmonics vastly increases the intrinsic space of possible dynamical states. Fortunately, at least for the cases to be described the sensitivity to initial data seems weak.

An additional qualification which must be kept in mind is that the increased computational burden presented by the full quasi-geostrophic equations does not allow us to run the calculations for as long as we might really like. Hence some solutions that appear chaotic may, with time, eventually become steady. This socalled "pre-turbulence" (Sparrow, 1982) behavior can be expected on the parameter border between chaotic and steady behavior. Generally we allowed the numerical calculation to proceed to 500 and occasionally to 1,000 linear $e$-folding times in an effort to be reasonably certain in our characterization of the wave behavior. Nevertheless, the value of the boundary between aperiodic and steady behavior, for example, may be somewhat imprecise.

After all these warnings and qualifications, certain broad qualitative features appear from the detailed calculations described in section 3 . In these calculations the supercriticality of the system, $\Delta$, ranged from being very small $(\Delta=0.02)$ to a value of about four times the critical value of $F,(\Delta=45)$.

Our final conclusion is that the interval in $\gamma$ over which aperiodic amplitude behavior exists increases with increasing supercriticality. Rather than being a consequence of any delicacy of weakly nonlinear theory, chaotic, "Lorenzian" behavior appears robust and enhanced by higher supercriticality. For a fundamental wave which is square, i.e., whose half-wavelength is the channel width $\left(a^{2}=2 \pi^{2}\right)$, the boundary between steady and aperiodic behavior moves from $\gamma \sim 0.18$ for $\Delta$ $=0.02$ to $\gamma \sim 0.25$ for $\Delta=14$. (This represents an increase of more than an order of magnitude in the value of $r$ for which aperiodic behavior occurs.) The overall behavior of the solutions as a function of $\gamma$ and $\Delta$, as determined by our calculations, are described in the map shown in Fig. 1.

The parametric complexity of the dynamical behavior, found in weakly nonlinear theory, remains in evidence, at least for $O(1)$ values of the supercriticality. For $\gamma=0.2$ for example, integrations of the full equations with an adequate representation of the solution structure reveal isolated islands of steady state behavior (e.g., in the range $8 \leqslant \Delta \leqslant 10$ ), and isolated intervals of periodic behavior $(3 \leqslant \Delta \leqslant 5)$ both embedded in a zone of aperiodic behavior. For small $\Delta, \gamma=0.2$ has only steady solutions. However no period doubling phenomena was observed as $\Delta$ increased although this may be the result of the absence of a sufficient fineness in the parameter net used.

The amplitude scaling used in weakly nonlinear theory (1) ensures that steady solutions of (7), (8) for $A$ are equal to unity. If the same measuring scale is used for the amplitude of the fundamental wave of the full system our results show that this scaled amplitude $d e$ creases with increasing $\Delta$. Since the overall amplitude is proportional to $\Delta^{1 / 2}$ [i.e., (i)] this implies that as $\Delta$ increases the amplitude of the steady solutions increases, but less rapidly than $\Delta^{1 / 2}$. In section $2 c$ this result is explained as a nonlinear "stiffening" of the system and can be accurately forecast by a truncated representation of the solution.

\begin{tabular}{|c|c|c|c|c|c|c|c|}
\hline & 05 & .12 & .14 & .17 & .2 & $\begin{array}{l}.23 \\
.25\end{array}$ & .3 \\
\hline $.02 t$ & & $\left(\begin{array}{l}p \\
p\end{array}\right)$ & $\left(\begin{array}{l}P \\
P\end{array}\right)$ & (AP) & $\left(\begin{array}{l}5 \\
5\end{array}\right)$ & & \\
\hline $1+$ & & $\left(\begin{array}{l}\mathrm{P} \\
\mathrm{P}\end{array}\right)$ & & & $\left(\begin{array}{c}A p \\
A p\end{array}\right)$ & $\left(\begin{array}{l}5 \\
5\end{array}\right)$ & \\
\hline $2 f$ & & & & & $\left(\begin{array}{l}\left(L^{\circ}\right. \\
A D\end{array}\right)$ & & \\
\hline $3-$ & & $\left({ }_{\mathrm{P}}^{\mathrm{L} C}\right)$ & & & $\left(\begin{array}{l}\alpha \\
\left(A^{\prime} C\right.\end{array}\right)$ & $\left(\begin{array}{l}5 \\
5\end{array}\right)$ & \\
\hline $5 f$ & & $\left(\begin{array}{ll}(2) \\
p\end{array}\right)$ & & $\left({ }_{A p}^{(L C}\right)$ & $\left(\begin{array}{c}1 \\
A-C\end{array}\right)$ & $\left(\begin{array}{l}s \\
s\end{array}\right)$ & \\
\hline $8-$ & & & & & $\left(\begin{array}{l}5 \\
A_{P}\end{array}\right)$ & & \\
\hline $\begin{array}{l}10 f \\
12\end{array}$ & & , & & & $\left(\begin{array}{l}5 \\
A\end{array}\right)$ & & \\
\hline 14 & $\left(\begin{array}{l}\text { LC } \\
P\end{array}\right)$ & $\left(\begin{array}{l}A p \\
A P p\end{array}\right)$ & & & $(\hat{A})$ & $\left(\begin{array}{l}A p \\
A p\end{array}\right)$ & $\left(\begin{array}{l}5 \\
5\end{array}\right)$ \\
\hline $20-$ & & & & & 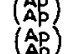 & & \\
\hline $45-$ & & & & & $\left(\begin{array}{c}A p \\
A p\end{array}\right)$ & & \\
\hline
\end{tabular}

FIG. 1. Map of the solution type in the $\gamma-\Delta$ plane. Points marked $S$ correspond to solutions observed to become eventually steady. The points marked $A_{p}$ refer to solutions which are aperiodic. Points marked $\mathbf{P}$ refer to solutions which become periodic in which the amplitude of the fundamental passes through zero. Points marked LC refer to solutions which are periodic for which the amplitude is always different from zero. These two solutions are also referred to in the text as the $E>0$ and $E<0$ limit cycles respectively. The calculations shown here were done for the case where the fundamental wave had $a^{2}=2 \pi^{2}$. Two labels are shown for each parameter value. The upper label refers to calculations employing the full representation of the solution while the lower label refers to calculations with the representation of the wave truncated to a single harmonic component. 
The role of the higher harmonics of the fundamental wave is observed to be a rather subtle one, at least for the supercriticalities studied. We observed (see Fig. 1) that the truncated system, consisting only of the fundamental wave and the mean flow, often predicted aperiodic behavior for those parameter values where the full system predicts steady or periodic solutions. A series of numerical experiments with various altered forms of the fundamental equations (see sections 2 and 3) leads us to believe that the most important effect of the higher zonal harmonics is the manner in which they change the meridional structure of the fundamental wave and the result which that altered meridional structure has on the structure of the evolving mean flow. This indirect stabilization effect of the higher harmonics is overwhelmed at higher supercriticality where both the full and truncated systems become aperiodic.

The calculation at $\Delta=45$, i.e., at about four times the critical value, presents a situation in which six waves present in the calculation are unstable. The most unstable wave is the wave with half-wavelength of the fundamental. Nevertheless (see Fig. 20) our calculations show that the most unstable wave gives way, after a short initial interval of dominance, to the longer fundamental wave. This longer wave then dominates the solution except for periods of minor bursts of activity of the shorter waves in a manner that has previously been seen in weakly nonlinear theory (Pedlosky, 1981). These are the principal conclusions drawn from the calculations we have completed. They are described more completely below along with other interesting but more technical results.

\section{Formulation of the mathematical problem}

\section{a. The spectral model}

A spectral numerical method (similar to the one used by Boville, 1980, 1982) based on Fourier series has been used to integrate the quasigeostrophic equations (15). For the zonal channel domain considered, an appropriate spectral representation of the streamfunctions, in the lower and upper layers, which satisfies the boundary conditions, i.e.,

$$
\begin{gathered}
\psi\left(x+\frac{L_{x}}{L}, y\right)^{\prime}=\psi(x, y) \\
\frac{\partial \psi}{\partial x}=0, \quad \int_{0}^{L_{x} / L} \frac{\partial \psi}{\partial y} d x=0 \quad \text { on } y=0,1,
\end{gathered}
$$

has been chosen as

$$
\psi=\sum_{m} \sum_{n} A_{m n} e^{i m k x} \sin n \pi y+\sum_{p} A_{0 p} \cos p \pi y
$$

with $k=2 \pi L / L_{x}, A_{m n}=A_{-m n}^{*},|m| \leqslant M$ with $m \neq 0$, $1 \leqslant n \leqslant N$ and $1 \leqslant p \leqslant P$. An asterisk denotes complex conjugation. The wavelength $L_{x}$ is of the fundamental wave (which corresponds to $m=1, n=1$ ). In this study we have chosen $L_{x}=2 L$ which means that the fundamental is square. In (19) $A_{m n}$ and $A_{0 p}$ designate the Fourier components respectively of the wave field and of the zonal flow correction (which is the component of $\psi$ independent of $x$ ). The wave field involves the fundamental and its harmonics.

Two sets of simulations corresponding to different values for $M$ and $N$ have been run. The first set concerns the so-called "truncated system" which involves only the fundamental for the wave field and corresponds to $M=N=1$. The second set concerns the socalled "full system" where the wave field is represented by the fundamental wave and its harmonics. In this case four or eight (when specified) zonal harmonics and eight meridional harmonics have been considered, which corresponds to $M=4$ or 8 and $N=8$. For both systems, the spectral representation of the zonal flow correction contains 24 components $(P=24)$. In most of the experiments carried out the fundamental is the only unstable wave and when it is not the case, ( $\Delta$ $\geqslant 16$ ), the fundamental becomes rapidly the dominant one. The harmonics are generally stable and from the numerical results their amplitudes are several orders of magnitude lower than the fundamental one. The calculated Fourier spectra (discussed in section 3) fall off very rapidly from the fundamental and display a very negligible fraction of energy retained in the latter harmonics. This has encouraged our belief in the adequacy of the representation used for the wave field.

Substituting the spectral representation (19) in Eqs. (15) yields a coupled set of equations for the time evolution of the spectral coefficients $A_{m n}$ and $A_{0 p}$. These equations have been integrated in time using a leapfrog differencing scheme for the advective terms, with application of a Robert filter (Asselin, 1972) to control and damp out the computational mode, while an implicit trapezoidal scheme (Kurihara, 1965) was used for the dissipation terms. The nonlinear Jacobian terms have been evaluated by using appropriately a spectral transform method introduced by Orszag (1971).

\section{b. Equations in Jacobian form}

In the results reported here, the fundamental wave, which is generally the dominant wave, displays in many cases a different behavior whether the effects of higher harmonics are taken into account or not. These effects are involved in the nonlinear interaction terms. In order to understand the role of the higher harmonics and what aspects of the dynamics produce these changes, several test simulations were run in each of which some specific contributions to the nonlinear interactions terms, involved in the fundamental wave and the zonal flow correction equations, were arbitrarily deleted.

More precisely, the nonlinear Jacobian terms in- 
volved in (15) are expanded as the streamfunction (19) in a Fourier series, i.e.,

$$
J=\sum_{m} \sum_{n} J_{m n} e^{i m k x} \sin n \pi y+\sum_{p} J_{0 p} \cos p \pi y
$$

with $|m| \leqslant M$ and $\dot{m} \neq 0,1 \leqslant n \leqslant N, 1 \leqslant p \leqslant P$ and $J_{m n}=J_{-m n}^{*}$. Attention has been focused on $J_{11}$, the nonlinear term involved in the equation for the time evolution of the amplitude of the fundamental wave $\left(A_{11}\right)$ and $J_{0 p}(1 \leqslant p \leqslant P)$ the nonlinear terms involved in the equation for the time evolution of the zonal mean flow components $\left(A_{0 p}\right)$. Here $J_{11}$ is the sum of three contributions: $J_{11}^{a}$, which is the interaction of the fundamental with the zonal flow; $J_{11}^{b}$, which is the interaction with the zonal flow of the waves with the same zonal wavenumber as the fundamental but with higher meridional wavenumber and $J_{11}^{c}$, the wavewave interactions involving waves with a higher zonal wavenumber than the fundamental. Also $J_{0 p}$ is the sum of three contributions which are $J_{0 p}^{a}$ the self-interaction of the fundamental wave, $J_{0 p}^{b}$ the wave-wave interaction involving waves with the same zonal wavenumber as the fundamental but excluding its self-interaction and $J_{0 p}^{c}$ the wave-wave interaction involving waves with higher zonal wavenumber.

In the test simulations reported below, the effects of these different contributions on the behavior of the fundamental wave have been evaluated.

\section{c. The truncated system}

In the limit of weakly nonlinear theory only the interaction of the fundamental wave with the mean flow is dynamically significant. Thus, in that limit there is a natural truncation of the solution representation in terms of a single harmonic. It is natural to wonder how well such a representation works at higher supercriticality, especially as the results to be described indicate that the energy in the higher harmonics is usually small (section 3) although not altogether negligible at larger $\Delta$.

In discussing the truncated system it is useful to consider the equations for the barotropic and baroclinic components of the streamfunction, i.e.,

$$
\left.\begin{array}{l}
\psi_{B} \equiv\left(\psi_{1}+\psi_{2}\right) / 2 \\
\psi_{T} \equiv\left(\psi_{1}-\psi_{2}\right) / 2
\end{array}\right\}
$$

in terms of which (15) becomes

$$
\begin{aligned}
\left(\frac{\partial}{\partial t}+\frac{r}{2}\right) q_{B}+ & U_{T} \frac{\partial}{\partial x} \nabla^{2} \psi_{T}+J\left(\psi_{B}, q_{B}\right)+J\left(\psi_{T}, q_{T}\right)=0 \\
\frac{\partial q_{T}}{\partial t}+\frac{r}{2} \nabla^{2} \psi_{T}+ & U_{T} \frac{\partial}{\partial x}\left(\nabla^{2} \psi_{B}+2 F \psi_{B}\right) \\
& +J\left(\psi_{B}, q_{T}\right)+J\left(\psi_{T}, q_{B}\right)=0, \quad(22 \mathrm{a}, \mathrm{b})
\end{aligned}
$$

where

$$
\begin{aligned}
U_{T} & =U_{s} / 2=U_{1}=-U_{2} \\
q_{B} & =\nabla^{2} \psi_{B} \\
q_{T} & =\nabla^{2} \psi_{T}-2 F \psi_{T} .
\end{aligned}
$$

We consider a formal solution to (15) of the form

$$
\begin{aligned}
& \psi_{B}=\tilde{A} e^{i k x} \sin l y+* \quad \equiv \varphi_{B} \\
& \psi_{T}=\tilde{B} e^{i k x} \sin l y+*+\Phi(y, t) \equiv \varphi_{T}+\Phi
\end{aligned}
$$

where 1 is an integral multiple of $\pi$.

Note that the only mean flow correction occurs for the baroclinic portion of the flow. Again an asterisk denotes complex conjugation.

Since for this truncation

$$
\begin{aligned}
J\left(\psi_{B}, q_{B}\right) & =0 \\
J\left(\psi_{T}, q_{T}\right) & =J\left(\varphi_{T}, \Phi_{y y}-2 F \Phi\right)-J\left(\Phi,\left(a^{2}+2 F\right) \varphi_{T}\right) \\
= & \frac{\partial \varphi_{T}}{\partial x} \cdot \frac{\partial}{\partial y}\left\{\Phi_{y y}+a^{2} \Phi\right\} \\
J\left(\psi_{B}, q_{T}\right)= & -\left(a^{2}+2 F\right) J\left(\varphi_{B}, \varphi_{T}\right) \\
& +\frac{\partial \varphi_{B}}{\partial x} \cdot \frac{\partial}{\partial y}\left\{\Phi_{y y}-2 F \Phi\right\} \\
J\left(\psi_{T}, q_{B}\right)= & -2 F J\left(\varphi_{T}, \varphi_{B}\right)+a^{2} \frac{\partial \varphi_{B}}{\partial x} \cdot \frac{\partial \Phi}{\partial y}(25 \mathrm{a}, \mathrm{b}, \mathrm{c}, \mathrm{d})
\end{aligned}
$$

while

$$
J\left(\varphi_{B}, \varphi_{T}\right)=i k l \sin 2 l y\left(\tilde{A} \tilde{B}^{*}-\tilde{A}^{*} \tilde{B}\right),
$$

it follows that the equations for $\tilde{A}, \tilde{B}$ and $\Phi$ may be written

$\frac{\partial}{\partial t}\left\{\frac{\partial^{2} \Phi}{\partial y^{2}}-2 F \Phi\right\}+\frac{r}{2} \frac{\partial^{2} \Phi}{\partial y^{2}}=i k l 2 F \sin 2 l y\left(\tilde{A} \tilde{B}^{*}-\tilde{A}^{*} \tilde{B}\right)$

$$
\begin{gathered}
\left(a^{2}+2 F\right) \frac{d \tilde{B}}{d t}+a^{2} \frac{r}{2} \tilde{B}-i k U_{T}\left(2 F-a^{2}\right) \tilde{A}+i k l \tilde{A} 2 \\
\times \int_{0}^{1} \sin 2 l y\left\{\frac{d^{2} \Phi}{\partial y^{2}}-\left(2 F-a^{2}\right) \Phi\right\} d y=0 \\
\frac{a^{2}\left(\frac{d}{d t}+\frac{r}{2}\right) \tilde{A}+a^{2} i k U_{T} \tilde{B}+i k l \tilde{B} 2}{\times \int_{0}^{1} \sin 2 l y\left(\frac{\partial^{2} \Phi}{\partial y^{2}}+a^{2} \Phi\right) d y=0}
\end{gathered}
$$

For small $\Delta$, weakly nonlinear theory shows that $\tilde{B}$ is $\mathrm{O}\left(\Delta^{1 / 2}\right)$ with respect to $A$ and a balance of the underlined terms in (29) results. All terms in (28) are of the same order $(\mathrm{O}(\Delta))$ with respect to $A$. An interesting departure from weakly nonlinear theory occurs in the 
final term in (28) representing the wave-mean flow interaction. For small $\Delta, 2 F=a^{2}+O(\Delta)$ and the final term in $(28),\left(2 F-a^{2}\right) \Phi$, is negligible. Hence for small $\Delta$, the advection of barotropic relative vorticity by the baroclinic zonal flow correction essentially cancels the advection by the barotropic wave of the "thickness" portion of the zonal flow potential vorticity correction. This cancellation leaves only the interaction of the barotropic wave with the relative vorticity of mean flow correction as the sole wave-mean flow stabilization effect for small supercriticality. For larger supercriticality $2 F-a^{2}$ will be $>0$ and the final term will usually be of the same sign as the relative vorticity, $\partial^{2} \Phi / d y^{2}$. This enhances the magnitude of the wave-mean flow interaction for large $\Delta$.

Note that for all $\Delta,(28)$ and (29) imply that $\tilde{A}$ and $\tilde{B}$ will remain out of phase by exactly $90^{\circ}$, a result which appears to be true even for the complete system when the results are scrutinized. The truncated system (27), (28) and (29) was run as a companion to the system using the complete spectral representation. In general the results of the truncated system (in which $\Phi$ is represented for the numerical calculations by the full cosine series of section $2 \mathrm{a}$ ) tended to overestimate the domain of chaotic behavior. However we found that when both systems indicated steady amplitude behavior as a final solution, the truncated solution accurately predicted the amplitude of the full solution.

It is therefore of some interest to examine the steady state form of (27), (28) and (29).

Writing

$$
\tilde{B}=i r \tilde{G} /\left(2 k U_{T}\right)
$$

we obtain, as a solution to (27) satisfying $\partial \Phi / d y=0$ on $y=0$ and 1 :

$$
\Phi=-\frac{F}{l U_{T}}[\sin 2 l y-2 l(y-1 / 2)] \tilde{A} \tilde{G}
$$

leading to

$$
\begin{gathered}
a^{2} \frac{r^{2}}{4} \tilde{G}-2 k^{2} U_{T}^{2} \Delta_{i} \tilde{A}+4 F k^{2} l^{2} \tilde{A}^{2} \tilde{G}\left[1+\frac{3}{2} \frac{\Delta_{i}}{l^{2}}\right]=0 \\
\tilde{A}-\tilde{G}-\frac{4 F l^{2}}{U_{T^{2} a^{2}}} \tilde{A} \tilde{G}^{2}\left(1-\frac{3}{4} \frac{a^{2}}{l^{2}}\right)=0
\end{gathered}
$$

where

$$
\Delta_{i}=F-a^{2} / 2 \text {, }
$$

i.e., $\Delta_{i}$ is the supercriticality with respect to the inviscid criteria $F=a^{2} / 2$ obtained from (5) when $r=0$. If we write, in analogy with (1)

$$
\begin{aligned}
& \tilde{A}=\frac{\Delta_{i}^{1 / 2} U_{T}}{a l} A \\
& \tilde{G}=\frac{\Delta_{i}^{1 / 2} U_{T}}{a l} G,
\end{aligned}
$$

we obtain

$$
\begin{aligned}
& \frac{a^{2} r^{2}}{8 \Delta_{i} k^{2} U_{T}^{2}} G-A+A^{2} G\left(1+\frac{2 \Delta_{i}}{a^{2}}\right)\left(1+\frac{3}{2} \frac{\Delta_{i}}{l^{2}}\right)=0 \\
& A-G+2 \frac{\Delta_{i}}{a^{2}}\left(1+2 \frac{\Delta_{i}}{a^{2}}\right)\left(\frac{3}{4} \frac{a^{2}}{l^{2}}-1\right) A G^{2}=0
\end{aligned}
$$

The first term on the left hand side of (35) is $\mathrm{O}\left(\gamma^{2}\right)$ and for all the cases considered represents a negligible contribution to (35). If that term is ignored, a particularly simple result for $A$ results, namely

$$
A= \pm\left(1+\frac{3}{2} \frac{\dot{\Delta}_{i}}{l^{2}}\right)^{-1}
$$

As $\Delta_{i} \rightarrow 0$ the amplitude of the steady solution approaches 1 in agreement with weakly nonlinear theory. However when $\Delta_{i} / l^{2}$ is no longer negligibly small the overall amplitude increases less slowly than $\Delta^{1 / 2}$ by the factor $1 /\left(1+\frac{3}{2} \Delta_{i} / l^{2}\right)$. This represents a nonlinear "stiffening" of the system. For $2 F-a^{2} \neq 0$ the advection by the mean flow of the perturbation vorticity no longer cancels the wave advection of main flow thickness. This cancellation as $\Delta_{i} \rightarrow 0$ is the reason only the relative vorticity of the mean flow correction enters (7). For $\Delta_{i} / l^{2}=\mathrm{O}(1)$ this is no longer the case. We will discuss the details of the numerical results in section 3 . However we remark here that the estimate (37), based on the truncated system gives an excellent prediction of the wave amplitude when the complete calculation shows that solution becomes steady. What is not true is that the truncated system can predict the nature of the ultimate form of behavior. To predict the nature of the final state the complete dynamics is required. When the solution is captured by a steady equilibrium point the resulting simplified steady dynamics allows the truncated system to give a good description of the end state. From a conceptual point of view greater significance should be attached to the concept of nonlinear stiffening. We shall see that even for the time dependent states a stiffening is observed although the full system is required to accurately calculate the solution. In each case the stiffening is manifested by a reduction in the amplitude that would be expected on the basis of a naive extrapolation of the scaling appropriate to weakly nonlinear theory.

\section{d. Scaling used for the presentation of the numerical results}

As discussed in section 1, numerical calculations of this study were conducted in the parameter space of $F$ and $r$ along lines of constant $\gamma$ mainly in an attempt to focus on the new effects of nonlinearity, in contrast with the weakly nonlinear theory. The $\gamma$ and $\sigma$ are defined [see (18)] in terms of the fundamental wave in 
our spectral expansion, since it is the most unstable wave except for a single experiment $(\Delta=45)$.

Most of the numerical results reported below concern the time evolution of the scaled amplitude $A_{B}$ of the barotropic part of the fundamental, defined as

$$
\psi_{B}=\frac{\Delta^{1 / 2}\left(U_{1}-U_{2}\right)}{2 a \pi} A_{B}(t) e^{i k x} \sin \pi y+* .
$$

The development time $t$ is scaled by the linear $e$-folding time, and $A_{B}(t)$ is a complex number. Our numerical results revealed that, generally (mainly when $\Delta<20$ ), the ratio of the real part of $A_{B}$ to its imaginary part is constant for $\gamma$ and $\sigma$ fixed: this ratio is infinite when $\Delta \ll 1$, which means that $A_{B}$ is real, and is $\mathrm{O}(1)$ when $\Delta=5$. This means also that in most simulations the real and imaginary parts of $A_{B}$ go through zero simultaneously and this fact has been exploited for the presentation of the numerical results. The plotted amplitude $A$ corresponds to the modulus of $A_{B}$ with a sign equal to the sign of the real part. Note that this sign is also an indicator of the phase difference between the barotropic and the baroclinic part of the fundamental wave, which has been observed to be always $\pm \pi / 2$.

\section{Discussion of the numerical results}

Integrations of the spectral equations for the quasigeostrophic equations were run over a parameter range in $\gamma$ that embraced the interval over which Pedlosky and Frenzen (1980) found periodic, chaotic and steady solutions. The value of the supercriticality, $\Delta=F-F_{c}$, was increased from a small value, to establish a connection with the results of weakly nonlinear theory and to establish confidence in our numerical method. The largest value of $\Delta$ attained in the calculation was $\Delta$ $=45$, about four times the critical value of $F$ for a wave with zonal wavelength equal to twice the channel width, i.e., for $a^{2}=2 \pi^{2}$. In all calculations the longest possible mode had $a^{2}=2 \pi^{2}$ and except for $\Delta=45$ this longest wave was the linearly most unstable wave.

Figure 1 shows a table, or map, of the points in parameter space at which calculations were made. In this figure each point is labeled twice. The upper label refers to the numerical calculation with the full system while the lower label refers to the calculation done with the truncated system in which the wave field is truncated to a single zonal and meridional harmonic (the zonal flow correction is represented in each case by the full cosine series).

For small $\Delta$, e.g., $\Delta=0.02$, there is excellent agreement between our results and the results of weakly nonlinear theory. Figure 2 shows the amplitude oscillation at $\Delta=0.02, \gamma=0.12$. A periodic solution with a period equal to 24 linear $e$-folding times is observed in complete agreement with the calculations of Ped- losky and Frenzen. Similarly, at $\Delta=0.02, \gamma=0.14$ an oscillation of doubled period (not springing from a period doubling cascade of the solution at $\gamma=0.12$ ) is found, Fig. 3, again in complete agreement with the results of weakly nonlinear theory. At $\Delta=0.02, \gamma$ $=0.17$ aperiodic behavior is observed, shown in Fig. 4 , while at $\Delta=0.02, \gamma=0.2$ a steady solution is obtained. Pedlosky and Frenzen's results show that for small $\Delta$ the solution in fact becomes steady by $\gamma=0.18$.

As the map in Fig. 1 demonstrates, increasing the supercriticality extends the interval of chaotic behavior to larger $\gamma$ (Note, as previously remarked even at fixed $\gamma$ increasing $\Delta$ increases the value of $r$ ). For example at $\gamma=0.2, \Delta=1$, for which the weakly nonlinear solution is steady, Fig. 5a shows that the solution is now aperiodic. Figure $5 \mathrm{~b}$ shows the portrait of the same solution in the phase plane $(A, d A / d t)$ while Fig. 5c shows the Lorenz return map for the solution in which the absolute values of the $M$ th maximum in the solution are plotted against the $(M-1)$ st. The steep cusplike peak to the return map is a characteristic signature of chaotic motion normally associated with the existence of a strange attractor (Sparrow, 1982). The solution calculated here used eight zonal harmonics and eight meridional harmonics to represent the wave field.

For $\gamma=0.2$ the character of the solution changes in the neighborhood of $\Delta=2$ and appears to be sensitive to the number of zonal harmonics used in the calculation. When either the truncated system is used or a system involving only four zonal harmonics is used the solution is aperiodic. However the solution with eight zonal harmonics shown in Fig. 6 is periodic. This periodic solution differs however from the periodic solutions found at lower supercriticality. The amplitude now does not go through zero and has a well defined minimum. A similar periodic solution occurs at $\Delta=3$, $\gamma=0.12$ and at $\Delta=3, \gamma=0.2$ for both four and eight zonal harmonics in the solution representation, (see Fig. 7). However at $\gamma=0.25$ the solution rapidly tends to a steady state (Fig. 8). The appearance of this new periodic solution is strikingly similar to the form of the unstable limit cycles, found in weakly nonlinear theory (Pedlosky 1982). Two types of limit cycles were shown to exist at small $\Delta$ and small $\gamma$, e.g., the socalled $E>0$ cycle, similar to that shown in Fig. 2 which is a stable solution as $\Delta \rightarrow 0$ and the $E<0$ limit cycle which was shown to be unstable. We here speculate that the effect of nonlinearity is to stabilize the $E<0$ cycle which, over part of the parameter space, now becomes the realized solution.

Aside from the new form of the periodic solution for $\Delta=3, \gamma<0.25$, it is more important to emphasize the fact that increasing the supercriticality from $\Delta=1$ to $\Delta=3$ has produced a more regular solution. Whereas the solution was chaotic at $\gamma=0.2, \Delta=1$, it is periodic 


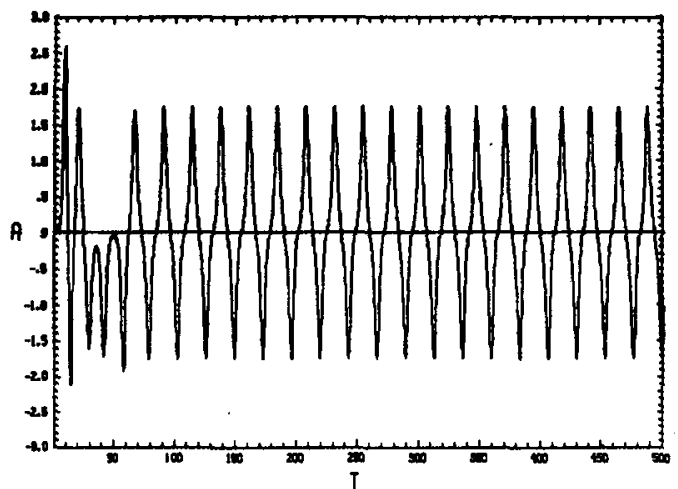

FIG. 2a. Time history of the periodic oscillation at $\gamma=0.12$, $\Delta=0.02$. In each case the amplitude shown is the real part of the $m=1, n=1$ barotropic mode as explained in section $2 \mathrm{~d}$.

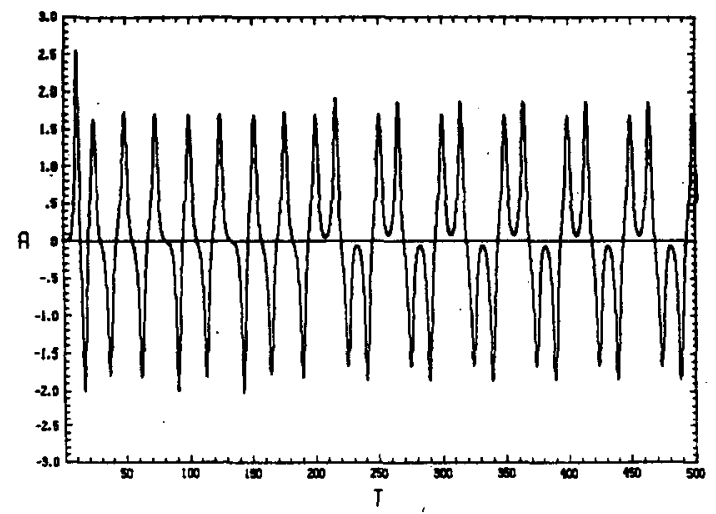

FIG. 3. Time history of the periodic oscillation at $\gamma=0.14, \Delta=0.02$.

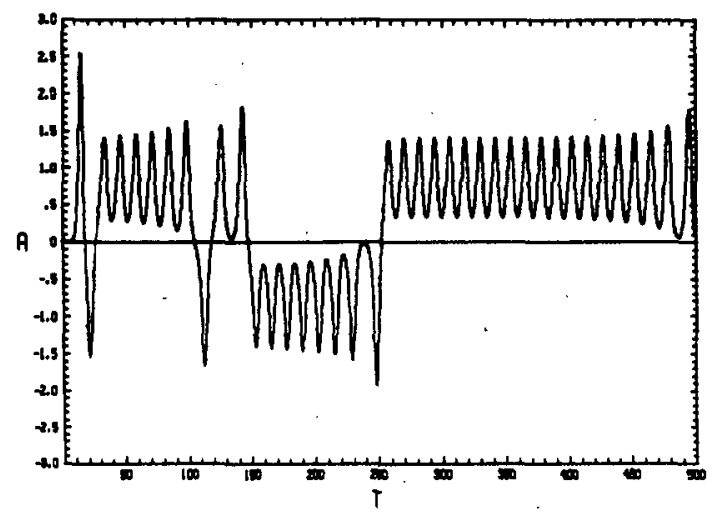

FIG. 5a. Time history of the aperiodic solution at $\gamma=0.2, \Delta=1$.

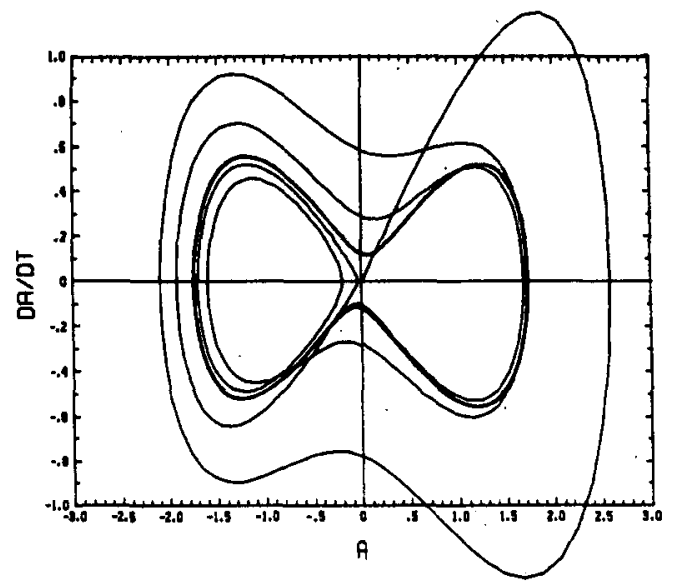

FIG. 2 b. Portrait of the same solution in the $A, d A / d t$ phase plane.

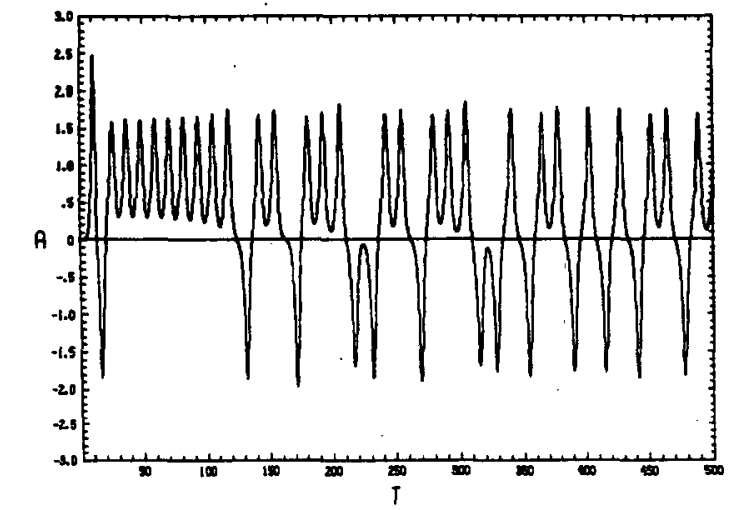

'FIG. 4. Aperiodic solution at $\gamma=0.17, \Delta=0.02$.

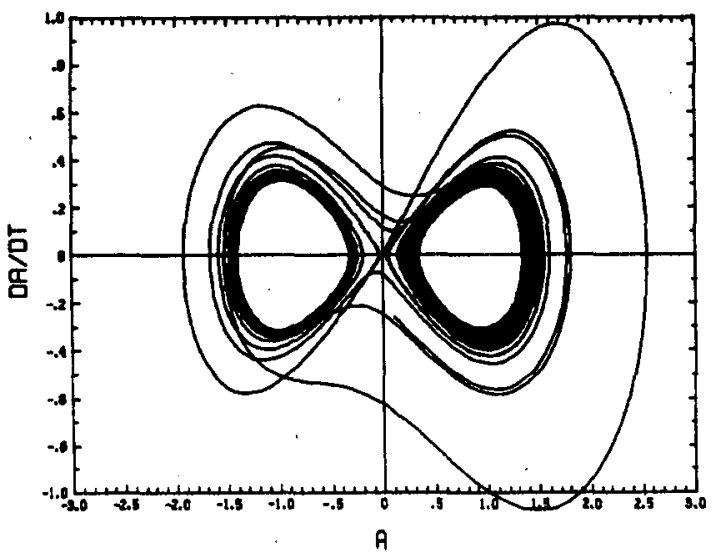

FIG. 5b. Portrait of the solution in the phase plane. 


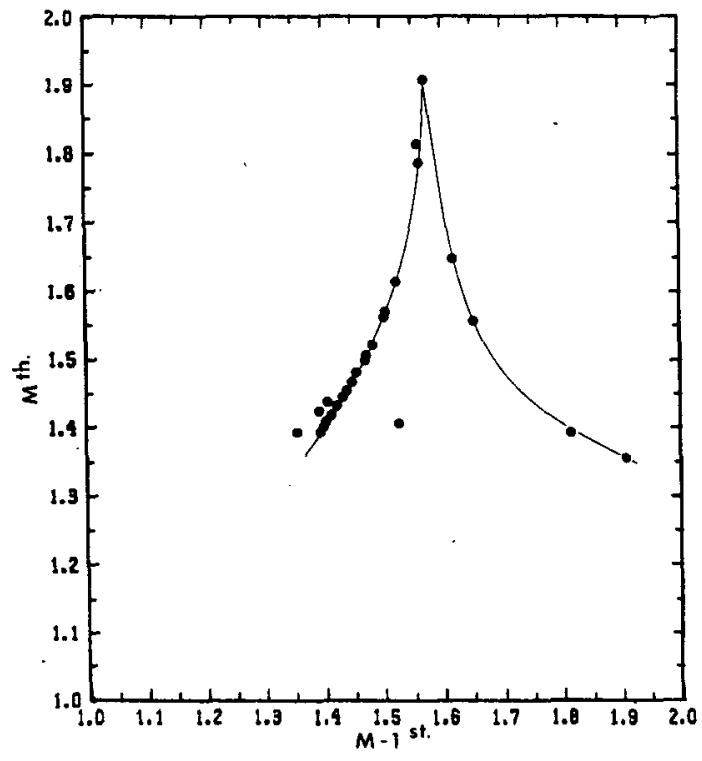

Fig. 5c. Lorenz return map illustrating the cusp vertex symptomatic of aperiodic behavior.

at $\gamma=0.2, \Delta=3$. The solution at $\gamma=0.2, \Delta=5$ is also periodic, again with the form of an $E<0$ limit cycle (Fig. 9). The phenomenon of nonlinear stiffening may be observed as both maximum amplitude and amplitude range are decreased (after removing the factor of $\Delta^{1 / 2}$ in the overall scaling) in moving from $\Delta=3$ to $\Delta=5$.

As $\Delta$ increases further, the solution becomes steady as shown in Fig. 10. The reemergence of a zone of steady amplitude solutions confounds the notion of a simple progression to turbulent or time dependent behavior with increasing $\Delta$. The amplitude of the steady solution $\sim 0.45$ is nearly exactly predicted by $(37)$ as indeed are the steady solutions at other parameter values and illustrates the nonlinear stiffening of the dynamics with increasing $\Delta$.

We wish to emphasize again that although the truncated model is a good estimator of the steady amplitude when it occurs, it is a poor predictor of the character of the realized solution. As Fig. 1 shows, the truncated system persistently predicts aperiodic behavior over a wide range of parameters for which the full system yields steady or periodic solutions. We will return to a more detailed diagnosis of this phenomenon shortly.

Proceeding to increase $\Delta$ at $\gamma=0.2$ produces aperiodic behavior at $\Delta=12,14,16$ and 20, e.g., Figs. $11 \mathrm{a}, \mathrm{b}$. The parameter mesh in Fig. 1 is admittedly coarse and it is quite possible that different dynamical behavior may occur in the intervals between the selected points. Generally however the aperiodic domain increases with increasing $\Delta$. Even with the aperiodic solutions the phenomenon of stiffening is observed as the amplitudes, after the removal of the scale factor $\Delta^{1 / 2}$, decrease with increasing $\Delta$.
At $\gamma=0.2, \Delta=20$ the modes $n=2, m=1$ and $m$ $=2, n=1$ are both weakly unstable in addition to the fundamental at $m=1, n=1$. Calculations were done to test the sensitivity of the results with different initial conditions for these modes. We found in the cases tested that these higher modes rapidly diminished in amplitude and the general behavior of the aperiodic solution for the fundamental $m=1, n=1$ mode was unaffected. This is not a proper test of the survivability of the most unstable mode, a point to which we return in our discussion below of the calculation at $\Delta=45$.

First, however, we wish to return to the nature of the differences of the solutions at lower $\Delta$ between the truncated and full systems. Figure 12a shows the solution for the truncated system for $\Delta=14$ and $\gamma=0.05$. The solution is periodic and looks very much like the periodic solutions achieved at small supercriticality. Figure $12 \mathrm{~b}$ shows the calculation of the solution of the full system at the same parameter value. Although the solution is once again periodic it is of a different type, i.e., of the $E<0$ limit cycle type mentioned above. The effect of the higher harmonics has been to exchange the type of realized limit cycle from the $E>0$ type, stable in weakly nonlinear theory, to the $E<0$ type. This effect of the truncation is even more dramatic at larger $\gamma$ where the effect of the truncation misrepresents a periodic solution as a chaotic one. Figure 13a shows the phase plane solution at $\Delta=5, \gamma=0.2$ while Fig. $13 \mathrm{~b}$ shows the phase plane solution at $\Delta=5, \gamma=0.2$ for the aperiodic solution predicted by the truncated system. We were astonished to realize that the limiting trajectory of the periodic solution of the full system formed almost exactly the inner boundary of the trajectories of the aperiodic solution as they circled the equilibrium point in the relevant half plane. There are two possibilities we can imagine. We have tentatively identified the periodic solution of the full system as an $E<0$ limit cycle stabilized at larger $\Delta$ by the presence of zonal harmonics. In the absence of the zonal harmonics this trajectory, as well as all others are destabilized producing chaotic behavior but remarkably this $E<0$ trajectory then forms the inner boundary of the aperiodic solution which the solutions may not penetrate. The second possibility is that the oscillation observed in the full system may in fact by very slowly decaying. Were this the case the inner boundary of the aperiodic solution would represent an unstable limit cycle. Solutions with the zonal harmonics then are found within the unstable limit cycle and wind down to the steady solution. This certainly occurs at $\Delta=10$, $\gamma=0.2$. However at $\Delta=3$ or $\Delta=5$ for $\gamma=0.2$ we believe the former description is more apt.

We do not pretend to have a full dynamical explanation for the changes in behavior and the appearance of new forms of periodic solutions or the role of the higher zonal harmonics in producing steady solutions. However we have tried to isolate what aspects of the dynamics are responsible. 


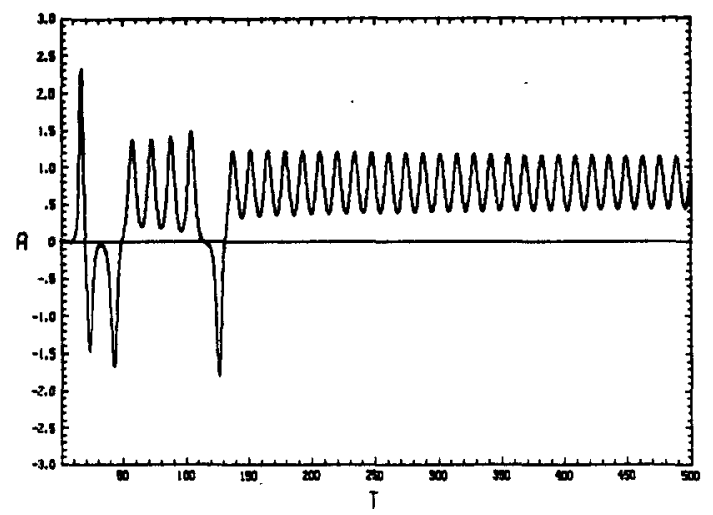

FIG. 6. Periodic solution at $\gamma=0.2, \Delta=2$.

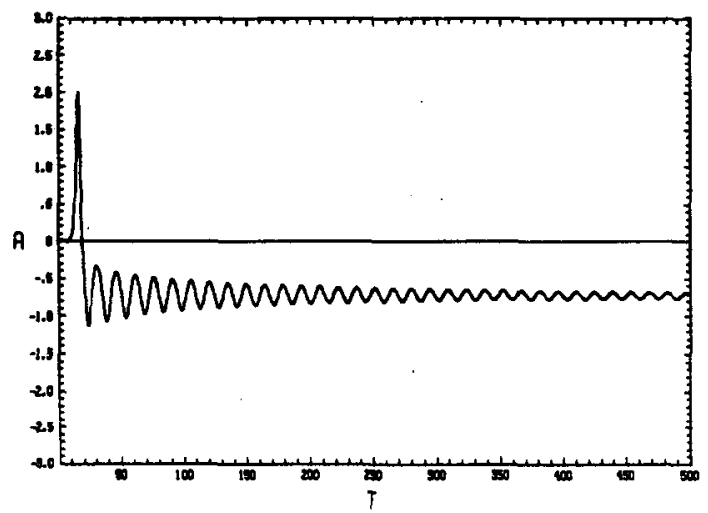

Fig. 8. Solution at $\gamma=0.25, \Delta=3$.

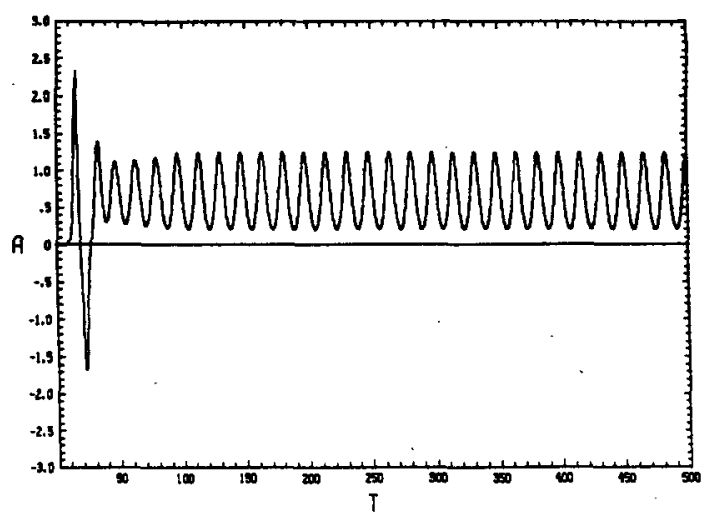

FIG. 7. Periodic solution at $\gamma=0.12, \Delta=3$.

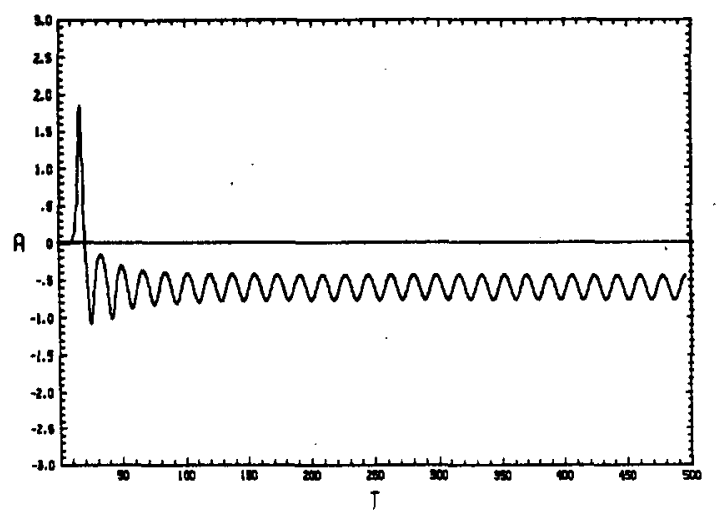

FIG. 9. Periodic solution at $\gamma=0.2, \Delta=5$.

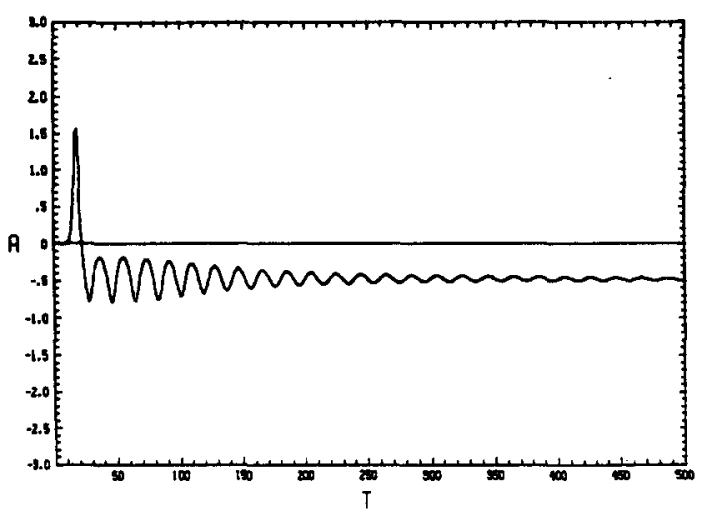

FIG. 10. Solution at $\gamma=0.2, \Delta=8$. 


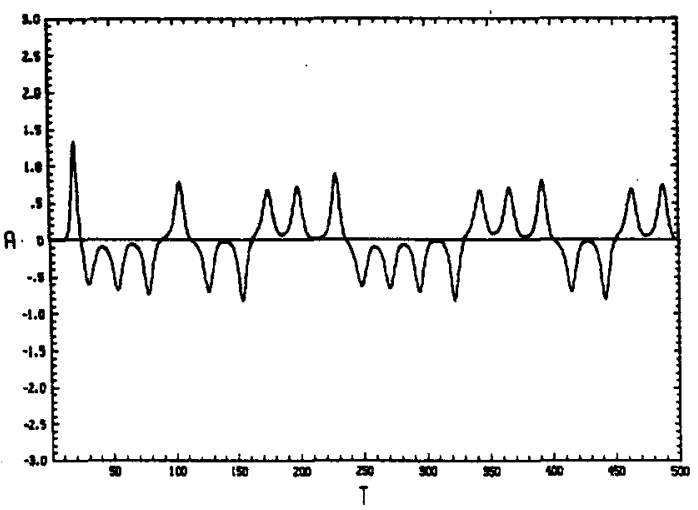

FIG. 11a. Aperiodic solution at $\gamma=0.2, \Delta=12$.

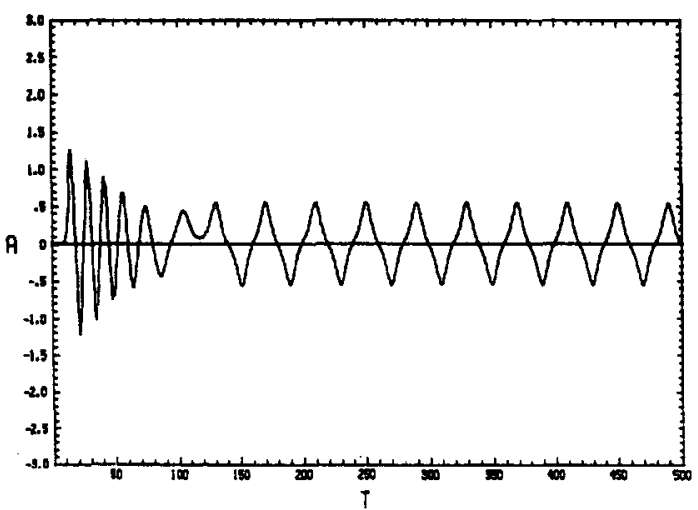

FIG. 12a. Periodic solution of the truncated system at $\gamma=0.05, \Delta=14$.

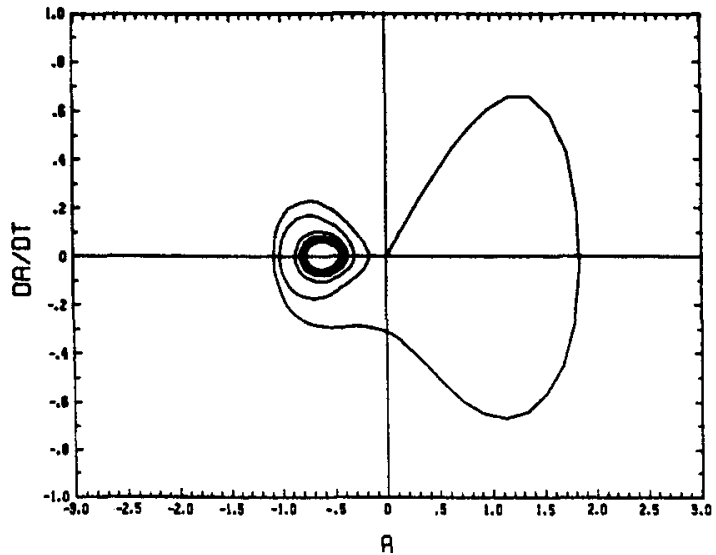

FIG. 13a. Phase plane portrait of the full solution at $\gamma=0.2, \Delta=5$.

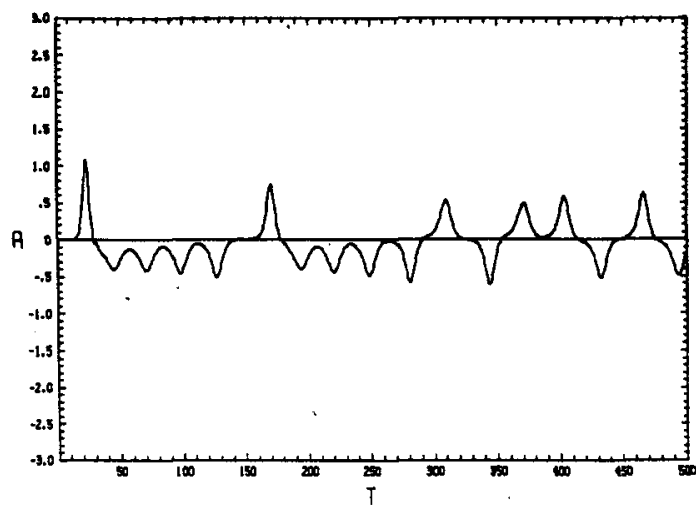

FIG. 11b. Aperiodic solution at $\gamma=0.2, \Delta=20$.

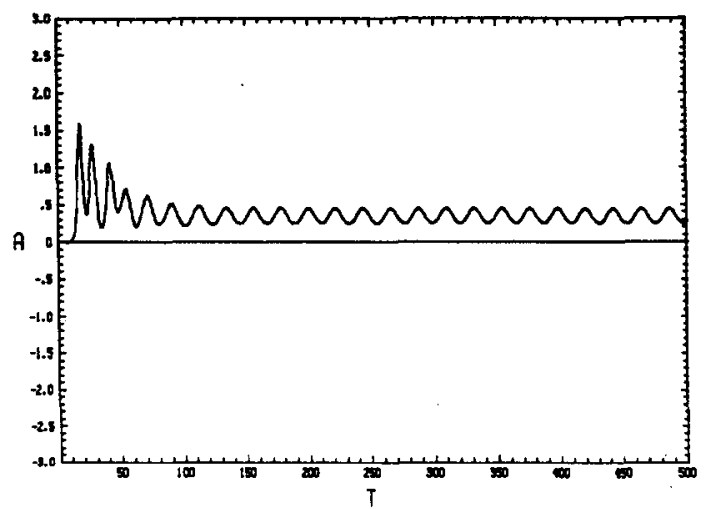

FiG. 12b. Periodic solution of the full system at $\gamma=0.05, \Delta=14$.

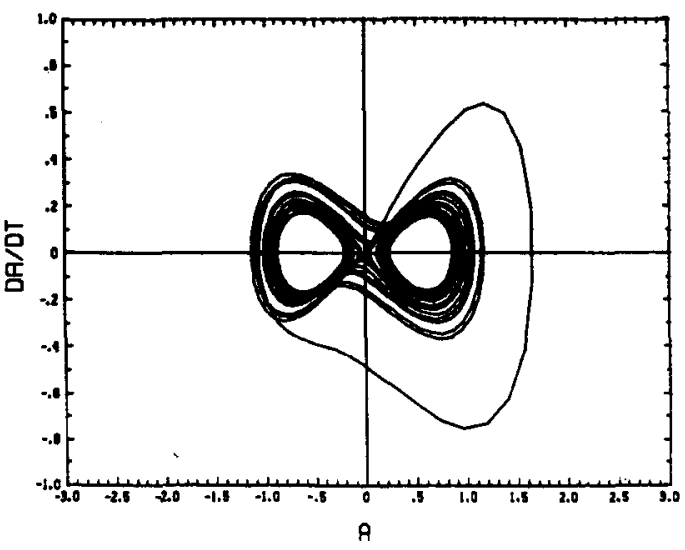

FIG. 13b. Phase plane portrait of the truncated system at $\gamma=0.2, \Delta=5$. 
We have focused on the case $\gamma=0.2, \Delta=5$ where the full system yields a periodic solution of a single sign for $A$ (the $E<0$ limit cycle) while the truncated system predicts aperiodic behavior as shown in Fig. $13 a, b$. In order to determine which aspects of the dynamics were responsible for the differing behaviors of the solutions a variety of test calculations were run in each of which some aspect of the full dynamics was arbitrarily deleted from the calculation. As described in section 2, the nonlinear interactions can be apportioned into three terms which contribute to the evolution of the fundamental wave. They are $J_{11}^{a}$, the interaction of the fundamental wave with the mean flow, $J_{11}^{b}$ the interaction with the mean flow of waves with the same zonal wavenumber as the fundamental but with higher meridional harmonics and $J_{11}^{c}$ which represents direct wave-wave interactions of higher zonal harmonics resulting in an alteration of the fundamental. Note that $J_{11}^{b}$ represents the effect of the altered meridional structure of the fundamental wave in its interaction with the mean flow. Similarly, we have noted that a similar partition of the contributions to the zonal flow correction equation can be effected. The three relevant contributions are $J_{0 p}^{a}, J_{0 p}^{b}, J_{0 p}^{c}$ which represent the self-interaction of the fundamental, the mutual interaction of waves with the same zonal wavenumber as the fundamental but excluding its selfinteraction and the mutual interaction of higher zonal harmonics respectively. In addition, of course, a similar partition can be made for any harmonic so that, for example, there will be terms in the equation for the higher meridional harmonics of the basic wave from wave-wave interactions as well as wave-mean flow interactions.

After a series of tests we found two cases which appeared to isolate the significant dynamical agent responsible for the changed character of the solution. Figure 14a shows a test calculation where in the equation for the fundamental wave only the interaction between the fundamental $(m=1, n=1)$ wave and the mean flow is retained and where, in the equation for the mean flow correction, we retain both the selfinteraction of the fundamental wave and the interaction of waves with the same zonal harmonic as the fundamental-excluding the self-interaction of the fundamental.

Hence this system represents only wave-mean flow interaction insofar as the fundamental is concerned but it fully represents how the altered meridional structure of the wave with the same zonal wavelength as the fundamental alters the zonal flow. Moreover, in this system the higher zonal harmonics continue to affect the flow insofar as they contribute to the evolution of the higher meridional harmonics of the basic wave. We see from Fig. 14a that this rather minimal representation of the amplitude dynamics reproduces the qualitative character of the full solution although the amplitude of the solution is smaller in the case where the full effect of wave-wave interaction is retained as shown in Fig. 9. Figure 14b shows a calculation with the same model as Fig. 14a with a single, slight change. As in Fig. 14a only $J_{11}^{a}$ is retained in the equation for the fundamental and only $J_{0 p}^{a}$ and $J_{0 p}^{b}$ are retained in the equation for the mean flow correction. However for the calculation shown in Fig. 14b the effect of the higher zonal harmonics of the fundamental are completely suppressed in the equations for the evolution of the higher meridional harmonics of the fundamental. Since these higher meridional harmonics can, in this system, only affect the dynamics by altering the mean flow it appears that the crucial role of the zonal harmonics is to allow a sufficiently accurate description of the meridional structure of the basic wave so that the correction to the zonal flow may be calculated correctly. That is, unless the $y$-structure of the basic wave takes into account wave-wave interactions, the zonal flow correction produced by the heat fluxes and Reynolds stresses of the basic wave will not be calculated accurately enough to get the wave-mean flow interaction of the fundamental correctly. This is a very subtle role for the zonal harmonics, a very indirect one but similar to a type already observed in a somewhat special problem in weakly nonlinear theory on the $\beta$-plane (Pedlosky, 1982) where this indirect role of the higher zonal harmonics also appears vital to the dynamics.

A somewhat more complex situation was encountered at $\Delta=10, \gamma=0.2$. At this parameter value the full system predicted an ultimately steady solution, Fig. $15 \mathrm{a}$, while the truncated system showed aperiodic behavior Fig. 15b. The same sequence of tests was performed as reported above, deleting various aspects of the nonlinear dynamics arbitrarily. As in the case reported on at $\Delta=5, \gamma=0.2$ the deletion of the direct effects of the wave-wave interactions of the higher harmonics on the dynamics of the fundamental and of the mean flow had qualitatively little result on the solution. It again became steady. That is, the deletion of $J_{11}^{c}$ and $J_{0 p}^{c}$ had no qualitative effect on the dynamics. When $J_{11}^{b}$ was deleted, i.e., when the effect on the fundamental of the interaction of its higher meridional harmonics was also deleted (i.e., with $J_{11}^{b}, J_{11}^{c}$ and $J_{0 p}^{c}$ were deleted) a limit cycle was obtained as shown in Fig. $15 \mathrm{c}$. When in addition only these same terms are neglected but the effects of the higher zonal harmonics are suppressed, insofar as they affect the dynamics of the higher meridional harmonics of the fundamental, aperiodic behavior once again appears Fig. 15d. Thus, once again, the qualitative nature of the dynamics is governed by the interaction of the basic zonal harmonic with the mean flow. The effect of the higher zonal harmonics is crucial but indirect. The higher zonal harmonics are largely important in accurately specifying the meridional structure of the basic wave and how 


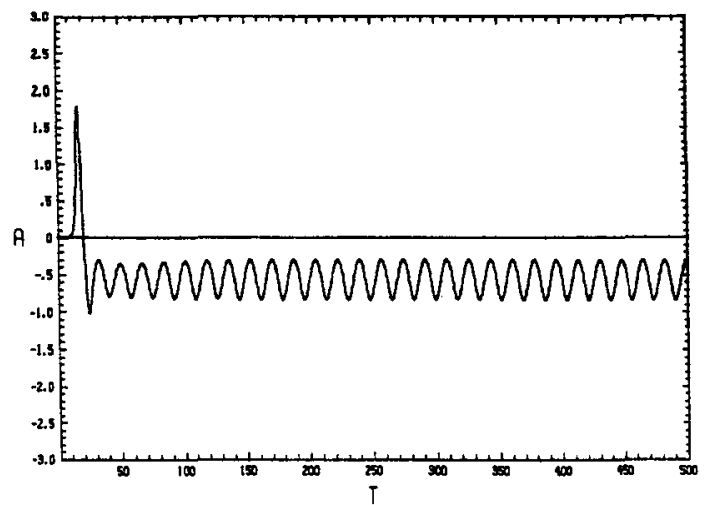

FIG. $14 \mathrm{a}$. The solution of the system retaining only $J_{11}^{a}, J_{0 p}^{a}$ and $J_{0 p}^{b}$ where the higher zonal harmonics affect the correction to the meridional structure of the basic wave.

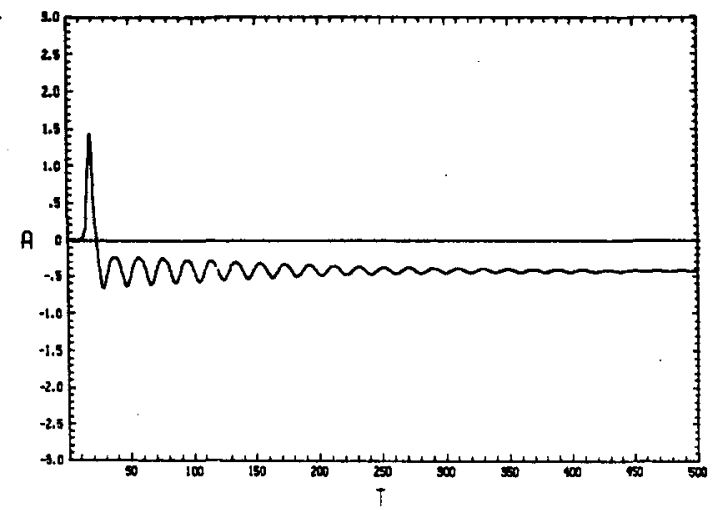

FIG. 15a. Steady solution obtained from the full system at $\gamma=0.2, \Delta=10$.

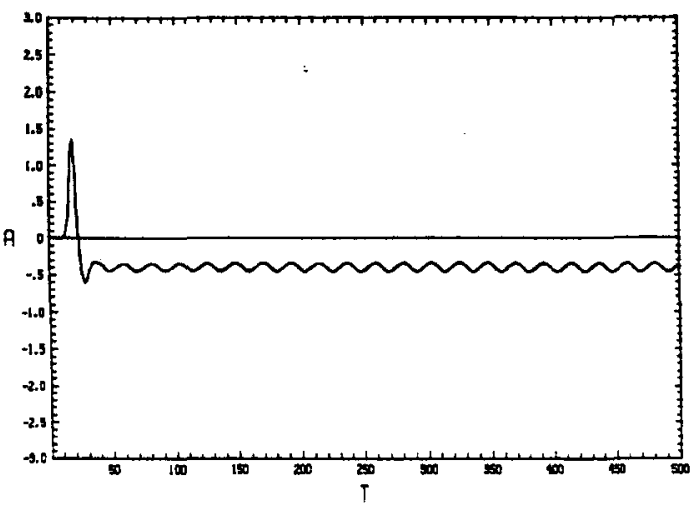

FIG. $15 \mathrm{c}$. Limit cycle obtained when $J_{11}^{b}, J_{11}^{c}$ and $J_{0 p}^{c}$ are neglected.

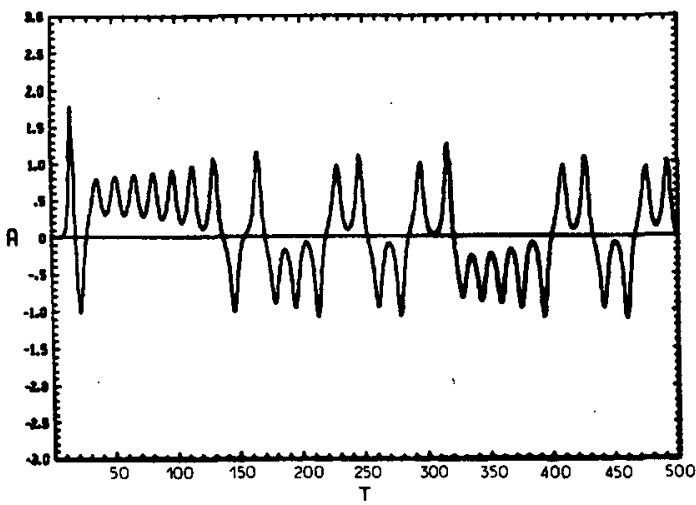

FIG. 14b. Solution of the system retaining the same nonlinear terms as in (a) but neglected the effect entirely of the higher zonal harmonics on the meridional structure of the basic wave.

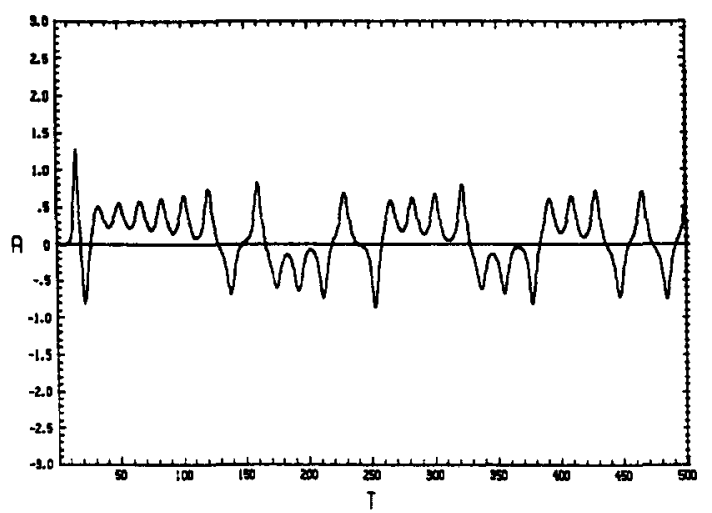

FIG. 15b. Aperiodic solution predicted by the truncated system at $\gamma=0.2, \Delta=10$.

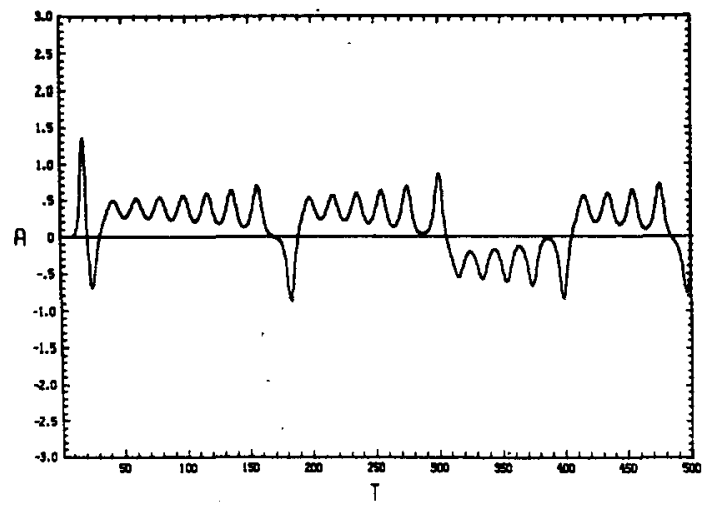

FIG. 15d. The effect of suppressing the effect of the higher zonal harmonics yields an aperiodic solution. 
this wave rectifies to alter the zonal flow. In this case $(\Delta=10, \gamma=0.2)$ the full system is "two steps" removed from the truncated system, i.e., steady as opposed to aperiodic. Suppression of the interaction of the altered meridional structure with the mean flow is enough to lift the system out of the steady state but it requires the complete suppression of the zonal harmonics to make it chaotic. Thus we conclude that for the cases studied at supercriticalities up to twice critical, that the dominant dynamics is overwhelmingly wave-mean flow interaction. Departures from weakly nonlinear theory enter primarily through the nonlinear alterations of the meridional structure of the basic wave. The accurate calculation of this alteration however requires the retention in the dynamics of the effects of the higher zonal harmonics.

These results are also consistent with our findings, which we do not present in detail here, that the Reynolds stresses in the fully developed waves remain small while the heat fluxes remain similar, though slightly reduced, to what would be expected from weakly nonlinear theory. Thus the dynamical processes are still largely those of weakly nonlinear theory although significant alterations in the amplitude and time dependence of the system are produced by the production of higher harmonics by frankly nonlinear processes.

Energy spectra have been estimated from the numerical results. It appeared to us more convenient to differentiate the energy spectrum for the wave field from the energy spectrum for the zonal flow correction. If $\psi^{\prime}$ and $\bar{\psi}$ designate the streamfunctions respectively for the wave field and for the zonal flow correction, energy spectra are estimated by

$$
\begin{aligned}
E_{w} & =\int_{0}^{L_{x} / L} d x \int_{0}^{1} d y\left[\left(\frac{\partial \psi^{\prime}}{\partial x}\right)^{2}+\left(\frac{\partial \psi^{\prime}}{\partial y}\right)^{2}\right] \\
& =\sum_{\substack{m \\
|m| \leqslant M \\
m \neq 0}} \sum_{\substack{n \\
m \neq n \in N}}\left(m^{2} k^{2}+n^{2} \pi^{2}\right)\left|A_{m n}\right|^{2}=\sum_{\kappa} A_{w}(\kappa)
\end{aligned}
$$

with $k=\left[\left(m^{2} k^{2}+n^{2} \pi^{2}\right) /\left(k^{2}+\pi^{2}\right)\right]^{1 / 2}$

$$
E_{z}=\int_{0}^{1} d y\left(\frac{\partial \bar{\psi}}{\partial y}\right)^{2}=\sum_{p} p^{2} \pi^{2} A_{0 p}^{2}=\sum_{\kappa} A_{z}(\kappa)
$$

with $\kappa=\left[p^{2} \pi^{2} /\left(k^{2}+\pi^{2}\right)\right]^{1 / 2}$. Spectra represent $A_{w}(\kappa)$ and $A_{z}(\kappa)$ as functions of $\kappa$.

Figures 16 and 17 show energy spectra for the cases $\Delta=1, \gamma=0.2$ and $\Delta=3, \gamma=0.2$ when eight zonal and eight meridional harmonics are considered. The first noticeable feature concerns the steepness of the calculated spectra. Energy spectrum for the zonal flow exhibits a small plateau and then falls off again very rapidly with a $\kappa^{-6}$ slope. Energy spectrum for the wave field falls off more rapidly with a $\kappa^{-7}$ mean slope. The fraction of energy retained in the latter harmonics is quite negligible with respect to the others. Besides the fundamental wave, the main components which emerge from the spectrum for the wave field correspond to the meridional mode $m=1, n=3$ and to the higher zonal modes $m=2, n=2$ and $m=3, n=1$. The other components are smaller and decay more and more rapidly as $k$ increases. This feature has encouraged our belief about the representation used for the streamfunction.

The energy spectra for the cases $\Delta=14, \gamma=0.2$ are shown on Fig. 18 and display the same features as with smaller $\Delta$, in particular concerning the steepness of the slopes.

Experiments were also carried out at much higher supercriticality at $\gamma=0.2, \Delta=45$, i.e., with $F$ about four times its critical value for the fundamental wave. At this parameter setting six of the harmonics are linearly unstable and, for the first time, one of the higher harmonics $m=2, n=1$ is the most unstable wave. Table 1 gives the (nondimensional) growth rates of the six modes.

Figure 19a shows the evolution of the solution for the case where, initially, the higher harmonics are zero. The solution is aperiodic. Note that the most unstable wave is not directly forced by the interaction of the fundamental with its higher harmonics and this component will remain zero if it is so initially. In this case we, a priori, expect a fairly sensitive dependence, then, on the initial data. Figure $19 \mathrm{~b}$ shows the solution for the same parameter setting for which the initial amplitudes of all the higher harmonics is initially nonzero. The differences on short times, $t<50$, is rather striking. As might be expected from linear theory the most unstable wave grows rapidly and initially dominates the spectrum. The initial spike in Fig. 19a for the $m=1$, $n=1$ mode is now absent being suppressed by the growth of the $m=2, n=1$ mode. However, shortly thereafter the amplitude level of the most unstable wave sinks to negligible levels not greatly different from other higher harmonics of the fundamental. After $300 e$ folding times (of the fundamental) and a sign reversal, the fundamental experiences a period during which its amplitude develops an oscillation of increasing magnitude symptomatic of a preparation for another sign flip. During this entire period, which lasts about 400 time units, the amplitudes of the higher harmonics hover near zero but never completely disappear. At $t$ $\sim 750$ the amplitude of the fundamental goes through zero and does not begin a new cycle of single signed oscillation for another 100 time units. In response to this temporary disappearance of the fundamental wave, the unstable higher harmonics spring to life. However there appears to be no simple relationship between the amplitudes of the harmonics during this burst of activity and their linear growth rates. For example, the harmonic $m=1, n=2$ which is ranked fifth in terms 


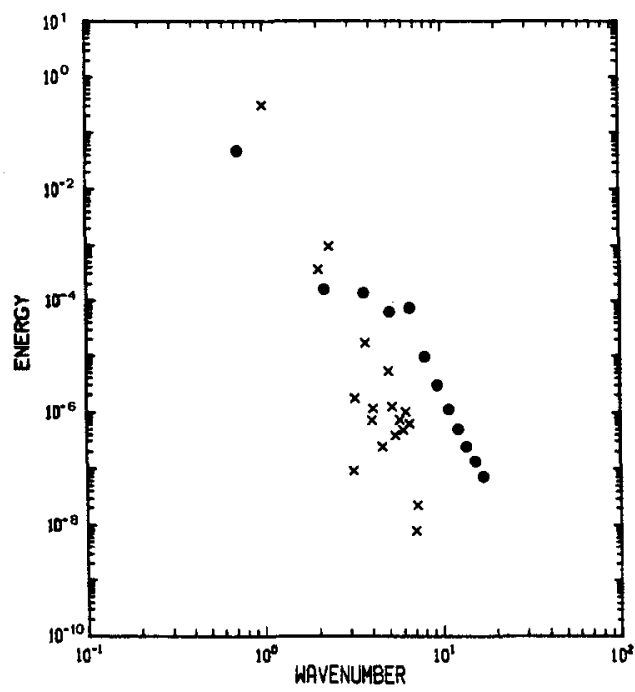

FIG. 16. Energy spectra for $\gamma=0.2, \Delta=1$ (eight zonal harmonics have been used in the simulation); $X$ and $\bullet$ symbols correspond to the spectrum respectively for the wave field and for the zonal flow correction.

of growth rate seems about as large as the $m=2, n$ $=1$ most unstable wave during this burst of activity.

The phenomenon of the disappearance of the most unstable wave and its replacement by a longer less unstable wave has been noted earlier, e.g., Hart (1981), Pedlosky (1981) and for $\Delta \ll 1$ can be explained in terms of weakly nonlinear theory. In that limit the dominance of wave-mean flow interaction provides the physical basis for the explanation. The most unstable wave saturates at an amplitude that leaves available energy for the more slowly growing, longer wave.

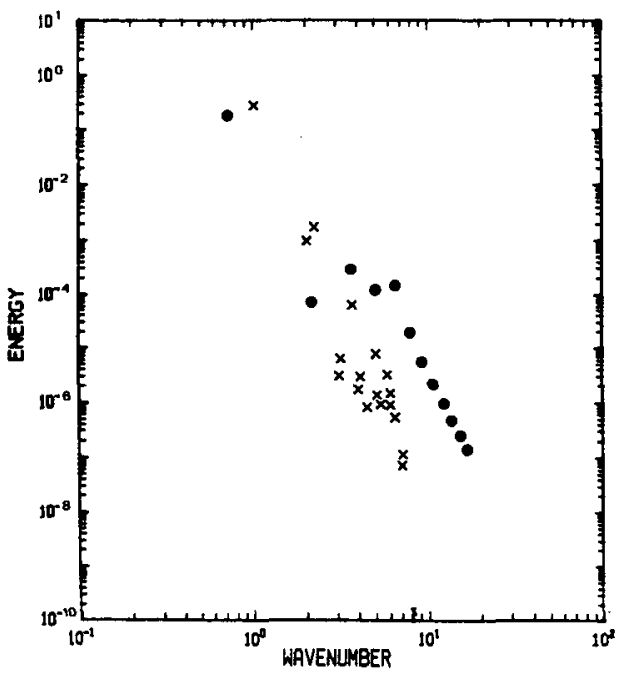

FIG. 17. As in Fig. 16 except for $\gamma=0.2, \Delta=3$.

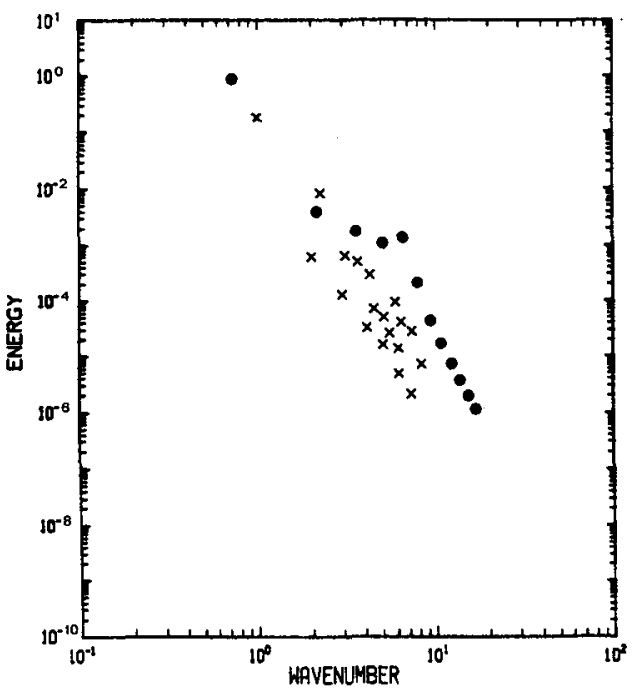

FiG. 18. As in Fig. 16 except for $\gamma=0.2, \Delta=14$.

As that wave grows it forces the amplitude of the most unstable wave to decrease. The process continues until the waves completely exchange roles as dominant and recessive waves. Clearly the same process occurs here at larger $\Delta$. Figure $19 \mathrm{c}$ shows the solution evolution at the same parameter setting when only four zonal harmonics are present in the solution representation. Nonetheless the qualitative nature of the solution remains the same. There are some interesting minor differences however to which we would like to draw the reader's attention. The amplitude of the fundamental, after achieving dominance, passes through zero more often in this case, and we note again that each time the fundamental either approaches zero closely (e.g., at $t \sim 625$ ) or passes through zero, a burst of activity in the higher unstable harmonics occurs.

The apparent discontinuous jump in the amplitude of the fundamental at $t \sim 225$ is an artifact of our representation. The reader will recall that for economy of presentation we have exploited the observed fact that generally the real and imaginary parts of the barotropic mode go through zero simultaneously (section $2 \mathrm{~d})$. Thus the plotted amplitude has been always the

TABLE 1. Nondimensional growth rates of the six modes.

\begin{tabular}{lll}
\hline \hline & $\begin{array}{c}\text { Mode } \\
(m, n)\end{array}$ & Growth rate \\
\hline 1 & $(1,1)$ & 2.107 \\
2 & $(1,2)$ & 1.382 \\
3 & $(1,3)$ & 0.070 \\
4 & $(2,1)$ & 3.212 \\
5 & $(2,2)$ & 1.947 \\
6 & $(3,1)$ & 1.652 \\
\hline
\end{tabular}




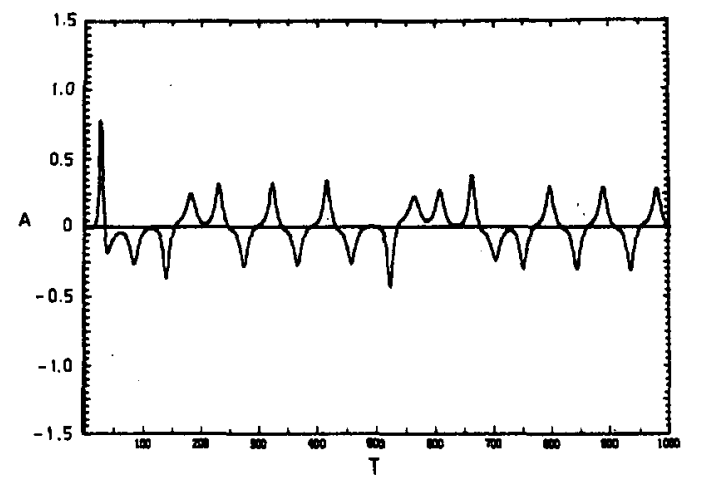

FIG. 19a. Solution at $\Delta=45, \gamma=0.2$ in which all harmonics except the fundamental are initially zero.

modulus of the barotropic mode and the sign has been the sign of the real part of the barotropic amplitude of the fundamental. At $t \sim 225$ we observe the unusual situation where the wave changed phase by $180^{\circ}$ without passing through zero.

We attempted to construct the Lorenz return map for the oscillation of the fundamental wave, and the result is shown in Fig. 19d. The map is dominated by the quasi regular period in which the size of each peak is increasing and the absence of a well resolved return map, as in Fig. 5, may be a signal that a much longer adjustment time will be required at higher supercriticality to establish definite Lorenz attractor behavior.

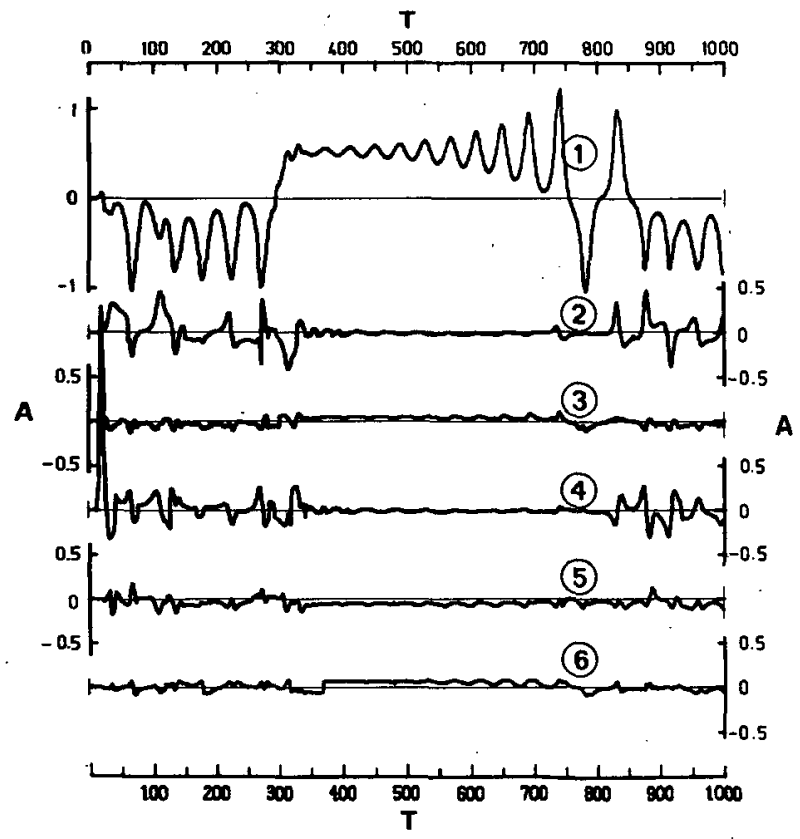

FIG. 19b. Solution, showing the evolution of the six linearly unstable harmonics for $\Delta=45, \gamma=0.2$ for the case where the initial amplitudes of the higher harmonics is nonzero. Eight zonal harmonics are used in the calculation. Labels refer to the harmonics identified in Table 1.

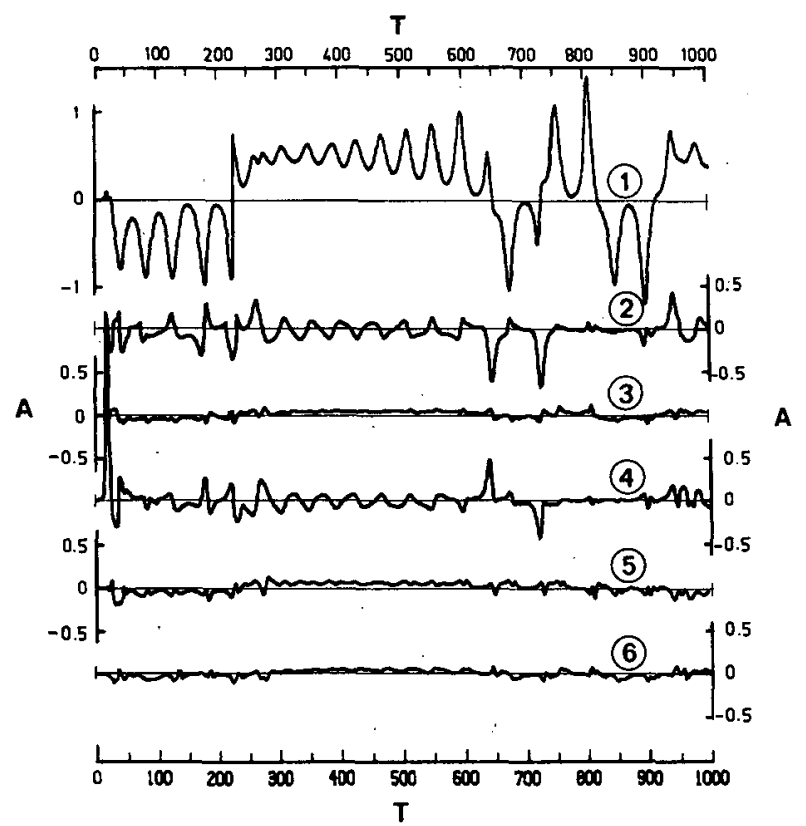

FIG. 19c. As in (b) except that four zonal harmonics are used to represent the wave field. In each case eight meridional harmonics are employed.

\section{Conclusions and discussion}

A series of numerical experiments with the two-layer quasi-geostrophic equations has examined the behavior of finite amplitude instability of a baroclinic flow with initially no meridional shear to disturbances on an $f$ plane. Friction acts equally on upper and lower layers.

The main results of our study are reviewed in the

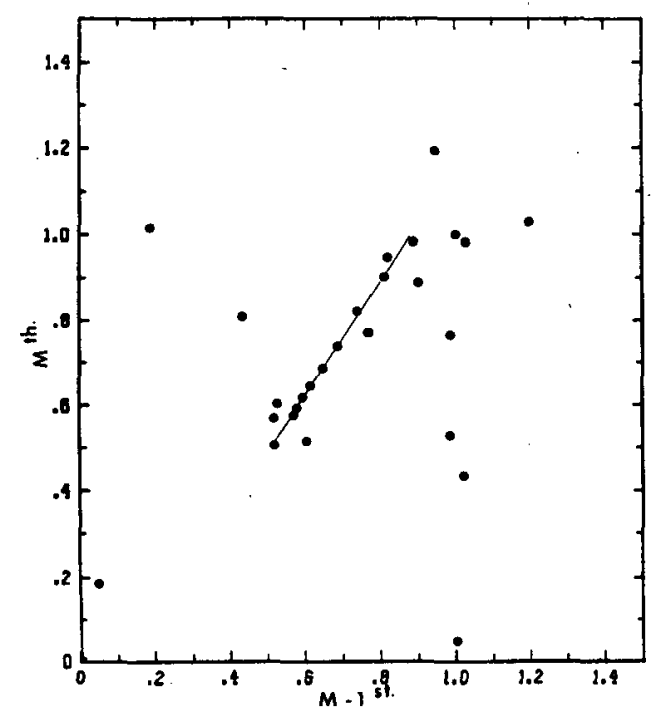

FIG. 19d. Lorenz return map for the oscillation of the fundamental shown in (b). 
introductory section of the paper, and so here we wish to limit ourselves to a few additional observations. For supercriticalities up to two to four times the critical value, the dynamics of the shear flow is complicated and subtle but its spatial structure remains fundamentally simple. The Fourier spectrum falls off very rapidly from the fundamental. Moreover certain dynamical properties found in weakly nonlinear theory, such as the "Lorenzian" strange attractor behavior, persist and characterize the motion. There is no sign that chaotic behavior is a particularly fragile aspect of weakly nonlinear theory.

However, we have noted some important qualitative changes with increasing supercriticality. These include the expansion of the domain of chaotic behavior and also the appearance of a new, apparently stable form of periodic motion. The dynamics also appears to retain its sensitive dependence on parameters. At higher supercriticality intervals of periodic, chaotic and steady wave behavior are interwoven. The accurate determination of the nature of the time behavior appears to require the representation of the interaction of the fundamental with its higher zonal harmonics, small though they may be.

The appearance of nonlinear stiffening of the system becomes apparent at higher supercriticality and is manifested by an increase of the wave amplitude with supercriticality at a rate slower than would be predicted on the basis of weakly nonlinear theory. It will be interesting to examine the nonlinear behavior of other models, e.g., on the $\beta$-plane, to discover if this is a general property.

The dominance of wave-mean flow interaction in the dynamics of the fundamental wave may be a property of the absence of horizontal shear in the basic state. This dominance is surely responsible for our observation of the preeminence of the longer wave over the most unstable wave in the case $\Delta=45$ in qualitative agreement with the weakly nonlinear theories of Hart (1981) and Pedlosky (1981).

There are a number of considerations which force us to remain tentative about our conclusions. As always we can never be sure that numerical calculations appearing to give chaotic behavior might not develop a more regular character were the calculation carried further. The point of view we have taken is 13 consider a run chaotic if it appears so for 500 to 1000 linear $e$-folding times. In fact to be certain about our characterization of the solution at $\gamma=0.25, \Delta=14$ the solution was run until $t=800$. One can observe that a long period of quasi-periodic behavior occurs before a transition to chaotic behavior becomes evident (Fig. 20). It is also true that we have felt constrained by the number of harmonics we have been able to include although the steepness of the calculated spectrum has encouraged our belief in the adequacy of the representation used.

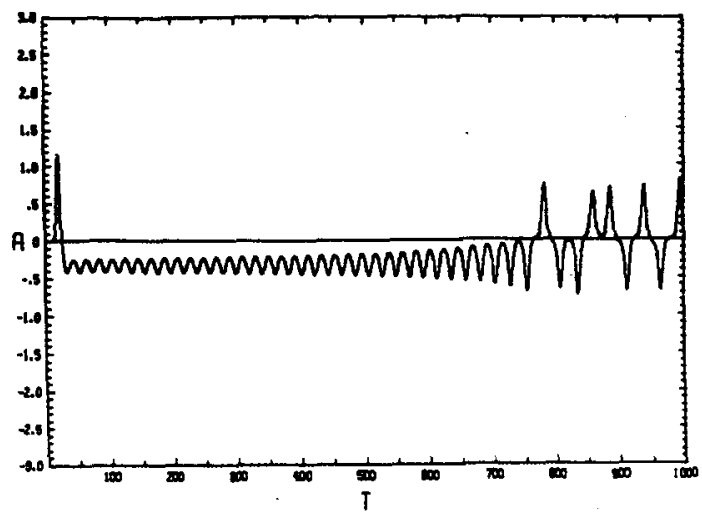

FIG. 20. Solution at $\gamma=0.25, \Delta=14$ showing the long time required before chaotic behavior becomes evident. At this value of $\Delta$ chaotic behavior occurs for $\gamma$ about 1.5 times the maximum value for chaotic behavior in weakly nonlinear theory.

Our long range goal is to carry out an investigation at even higher values of supercriticality to try to observe the transition from a highly structured, albeit very nonlinear wave system, to fully developed two-dimensional turbulence. Quite clearly the larger $\Delta$ becomes, the more harmonics we will eventually have to consider.

Our major source of concern remains with the physics and not the machinery of calculation. Investigations of weakly nonlinear systems have displayed a remarkable, in fact, stunning sensitivity to small changes in the physical nature of the problem whether it be the introduction of the $\beta$-effect, the presence of an upper free surface or the presence of horizontal shear. We confess to continued uncertainty in the generality of the results presented here with respect to changes in the physical structure of the basic problem.

Acknowledgments. The calculations reported here. were done at both the National Center for Atmospheric Research and the Centre de Calcul Vectoriel pour la Recherche. One of us (J.P.) is partially supported by a Grant from the Atmospheric Science Division of the National Science Foundation.

\section{REFERENCES}

Asselin, R., 1972: Frequency filter for time integrations. Mon. Wea. Rev., 100, 487-490.

Boville, B. A., 1980: Amplitude vacillation on an f-plane. J. Atmos. Sci., 37, 1413-1423.

- 1982: Strongly nonlinear vacillation in baroclinic waves. $J$. Atmos. Sci., 39, 1227-1240.

Drazin, P. G., 1970: Nonlinear baroclinic instability of a continuous zonal flow. Quart. J. Roy. Meteor. Soc., 96, 667-676.

, 1972: Nonlinear baroclinic instability of a continuous flow of a viscous fluid. J. Fluid Mech., 55, 577-588.

Hart, J. E., 1972: A laboratory study of baroclinic instability. Geophys. Fluid Dyn., 3, 181-209. 
- 1973: On the behavior of large-amplitude baroclinic waves. $J$. Atmos. Sci, 30, 1017-1034.

- 1981: Wavenumber selection in baroclinic instability. J. Atmos. Sci., 38, 400-408.

Kurihara, Y., 1965: On the use of implicit and iterative methods for the time integration of the wave equation. Mon. Wea. Rev., 93 , 33-46.

McWilliams, J. C., 1984: The emergence of isolated coherent vortices in turbulent flow. J. Fluid Dyn., 146, 21-43.

Orszag, S. A., 1971: Numerical simulation of incompressible flows within simple boundaries: 1: Galerkin (spectral) representations. Stud. Appl. Math., 50, 293-327.

Pedlosky, J., 1970: Finite amplitude baroclinic waves. J. Atmos. Sci., $27,15-30$.
1972: Limit cycles and unstable baroclinic waves. J. Atmos. Sci., 29, 53-63.

—, 1979: Geophysical Fluid Dynamics. Springer-Verlag, 624 pp.

- 1981: The nonlinear dynamics of baroclinic wave ensembles. J. Fluid Mech., 102, 169-209.

_- 1982: A simple model for nonlinear critical layers in an unstable baroclinic wave. J. Atmos. Sci., 39, 2119-2127.

amplitude baroclinic waves. J. Atmos. Sci., 37, 1177-1196.

Rhines, P. B., 1979: Geostrophic turbulence. Annual Review of Fluid Mechanics, 11, Annual Reviews, 401-441.

Salmon, R., 1982: Geostrophic turbulence. Topics in Ocean Physics, Soc. Italiana di Fisica, 30-78.

Sparrow, C., 1982: The Lorenz Equations: Bifurcation, Chaos and Strange Attractors. Springer-Verlag, 269 pp. 